\title{
QUASI-MINIMAL SEMI-EUCLIDEAN LAMINATIONS IN 3-MANIFOLDS
}

\author{
David GabaI
}

$\S 0$

\section{INTRODUCTION}

Thurston's hyperbolization conjecture [Th] asserts that a closed, atoroidal, irreducible 3-manifold with infinite fundamental group has a metric of constant negative curvature. A more modest form of the conjecture asks whether the fundamental group of such a manifold is negatively curved in the sense of Gromov [Gr]. (E.g. see $[\mathrm{Bu}],[\mathrm{Sch}],[\mathrm{Mol}],[\mathrm{MO}]$ or $[\mathrm{Ka}]$.$) A main result of this paper, the Ubiquity Theo-$ rem, provides a technique for addressing the group negative curvature conjecture. We use it in $[\mathrm{GK}]$ to show that an atoroidal 3-manifold with a genuine essential lamination has group negative curvature. (These manifolds appear to form a vast subset among the irreducible atoroidal 3-manifolds with infinite $\pi_{1}$. See the survey [G4].)

\footnotetext{
*Partially supported by NSF Grant DMS-9505253 and the MSRI
} 
In this paper the ubiquity theorem is used to derive new information about quasi least area semi-Euclidean laminations in 3-manifolds.

Gromov stated 6.8.S [Gr] that if $M$ is a compact manifold or finite simplicial complex whose fundamental group is nonnegatively curved, then there is a non constant least area conformal map $f: \mathbb{R}^{2} \rightarrow M$. Mosher and Oertel [MO] provided the first detailed proof (of a sharper version) in the context of finite 2-complexes. Recently Kleiner [Kl] showed that if $M$ is a closed Riemannian $n$-manifold such that $\pi_{1}(M)$ is non negatively curved, then there exists a branched Lipschitz conformal least area immersion $f: \mathbb{R}^{2} \rightarrow M$. If $n=3$, then $f$ is an immersion, though the induced metric on $\mathbb{R}^{2}$ may be incomplete.

In $\S 1$ we state basic definitions regarding immersed laminations and branched surfaces in 3-manifolds. Then we outline and discuss the proof of the main technical result of $[\mathrm{MO}]$ in the 3-manifold context. Their proof makes essential use of a theorem of Ghys and the Plante argument for immersed surfaces. In $\S 2$ we show how to extend $[\mathrm{MO}]$ to obtain our first main result.

Theorem 2.1. If $M$ is a closed non negatively curved 3-manifold, then there exists an immersed strongly least area, Euler characteristic 0, measured semi-Euclidean lamination $\lambda$. The induced metric on each leaf is complete.

Here $M$ has a triangulation $\tau$, and the various measurements of length and area are computed simplically. Strongly least area [MO] means that if $L$ is a leaf and $D$ is an embedded disc in the universal covering of $L$, then the induced map $i: D \rightarrow M$ is a least area map. Semi-Euclidean means that the set of leaves, conformally equivalent to the Euclidean plane, are of full measure and dense in $\lambda$.

By replacing immersed by branched immersed in the above statement, we obtain the exact translation of the main result of [MO] to 3-manifolds. The content of $\S 2$ is how to eliminate the branched points.

Our second main result states that if $k$ is a smooth simple closed curve in the irreducible group nonnegatively curved 3-manifold $M$, then either $k$ lies in a 3-cell, or $M$ is toroidal or among least area discs $D_{\alpha}$ of small isoperimetric ratio, $\operatorname{area}\left(D_{\alpha}\right)$ 
is approximately the geometric intersection number of $D$ and $k$. (By definition $M$ is toroidal if and only if $Z \oplus Z \subset \pi_{1}(M)$. By [CJ], [G2] the topology of closed irreducible toroidal manifolds is completely understood.) More precisely we have the following equivalent results.

Theorem 5.1. Let $k$ be a smooth simple closed curve in the closed irreducible Riemannian 3-manifold $M$. Then either $M$ is toroidal or $k$ is contained in a 3-cell or there exists a constant $C>0$ such that if $D$ is a least area disc with $\partial D \cap k=\emptyset$, then area $(D) \leq C(w r(\partial D, k)+$ length $(\partial D))$.

Ubiquity Theorem 5.2. Let $k \not \subset B^{3}$ be a smooth simple closed curve in the closed, atoroidal, irreducible 3-manifold $M$. There exists constants $K$ and $L$ such that if $D$ is a least area disc with $\partial D \cap k=\emptyset$ and length $(\partial D) / \operatorname{area}(D)<L$, then $w r(\partial D, k) / \operatorname{area}(D)>K$.

If $k$ is a simple closed curve in $M$, and $\alpha$ is a homotopically trivial curve in $M$ disjoint from $k$, then we define the wrapping number $\operatorname{wr}(\alpha, k)$ to be the minimal geometric intersection number between $k$ and all immersed discs $D_{\alpha}$ spanning $\alpha$.

Corollary 5.3. Let $\lambda$ be a quasi least area semi-Euclidean lamination in the closed atoroidal irreducible 3-manifold $M$. If $k \subset M-\lambda$ is a smooth simple closed curve, then $k$ lies in a 3 -cell.

Chapter $\S 3$ explains how to use cellulations of hyperbolic 3-manifolds by ideal polyhedra to obtain PL versions of various results known in the Riemannian world. In $\S 4$ we prove the Ubiquity Theorem in the PL category. Chapter $\S 5$ is devoted to proving the Ubiquity Theorem in the smooth category.

In $\S 6$ we give a very brief survey of progress on the Thurston geometrization conjecture.

Since the unresolved parts of Thurston's conjecture only concern orientable 3manifolds, we will assume that all 3-manifolds in this paper are orientable.

Readers Advisory. If you are interested in Ubiquity theorem, then skip directly to $\S 4$ or $\S 5$ and refer back as needed. Except for minor references to earlier stated 
definitions or figures, §3-6 are completely independent from the earlier chapters. To get right to the heart of the matter in the PL category, read 4.1-4.4 and then read the proof of the case, $M-k$ is hyperbolic. To read $\S 5$ one need only refer back to three self contained arguments of $\S 4$.

I would like to thank Igor Rivin for several key conversations about hyperbolic cellulations.

\section{Immersed Branched Surfaces and Laminations}

Definition 1.1. A generic immersed branched surface $B=\left(B^{*}, \phi\right)$ in a 3-manifold $M$ is a finite 2-complex $B^{*}$ which is mapped via $\phi$ into $M$ as follows. Each $x \in B^{*}$ has a neighborhood $U$ whose image appears as in Figures 1.1 a-d. Furthermore if $\phi(U)$ appears as in Figures $1.1 \mathrm{a}-\mathrm{c}$, then $\phi \mid U$ is an embedding. (Figures $1.1 \mathrm{a}-\mathrm{c}$ are the standard local models of a generic embedded branched surface [FO].) If $\phi(U)$ appears as in Figure $1.1 \mathrm{~d})$ then $U$ is homeomorphic to the 2-complex shown in Figure 1.1e) and $\phi \mid U$ fails to be an immersion only at $x$. Furthermore $\phi(U)$ has an arc of double points which starts at $\phi(x)$. The model shown in Figure 1.1 d) is the crossed maw discovered by Joe Christy [Ch]. The singularity is similar to the undrawable singularity of Poenaru [Po]. We assume that the various self intersections of $\phi$ are transverse and generic. Define the branch locus $\gamma(B)$ of $B$ to be the set of nonmanifold points of $B^{*}$, define a sector to be a component of $B^{*}-\gamma(B)$, and define $\partial B$ to be the union of free edges of $B^{*}$. We will often abuse notation by referring to the branch locus and boundary of $B$ as objects in $M$. We will also refer to generic immersed branched surfaces simply as branched surfaces. 


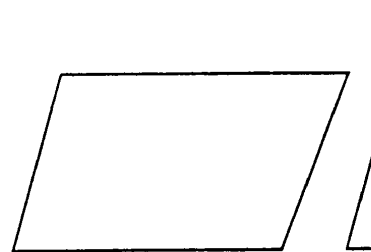

a)

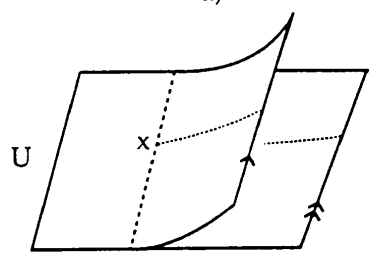

e)

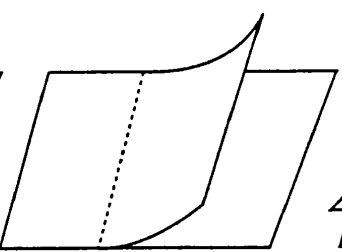

b)

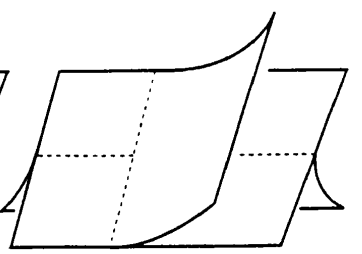

c)

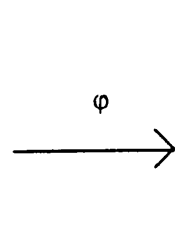

Figure 1.1

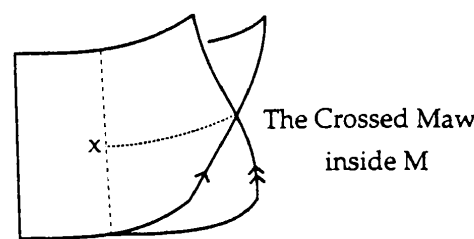

d)

Associated to the branched surface $B$ we define the normal neighborhood $\mathcal{N}(B)=$ $\left(\mathcal{N}\left(B^{*}\right), \phi\right)$ as follows. If $B^{*}$ is a locally finite union of embedded discs $D_{i}$, then define $\mathcal{N}\left(B^{*}\right)=\cup D_{i} \times[-1,1]$ modulo the equivalence relation, where $x \times[-1,1]$ is identified with $y \times[-1$,disjoint from $\partial \mathrm{E} 1]$, if $x=y \in B^{*}$. The identification is either the identity or $t \rightarrow-t$ depending on whether or not the local orientations on $D_{i}$ and $D_{j}$ agree when viewed inside of $M$. The singular immersion $\phi: B^{*} \rightarrow M$ induces a singular immersion $\phi: \mathcal{N}\left(B^{*}\right) \rightarrow M$. The mapping will be non immersive exactly along $\Gamma(B)$ the normal branched locus which we define to be $\pi^{-1}(\gamma(B))$ where $\pi: \mathcal{N}\left(B^{*}\right) \rightarrow B^{*}$ denotes the natural projection. See Figure 1.2. We identify $B^{*}$ with the 0 -section of $\mathcal{N}\left(B^{*}\right)$. The notation $\mathcal{N}(B)$ should not be confused with the similar notation $N(B)$ for fibred neighborhood of an embedded branched surface.

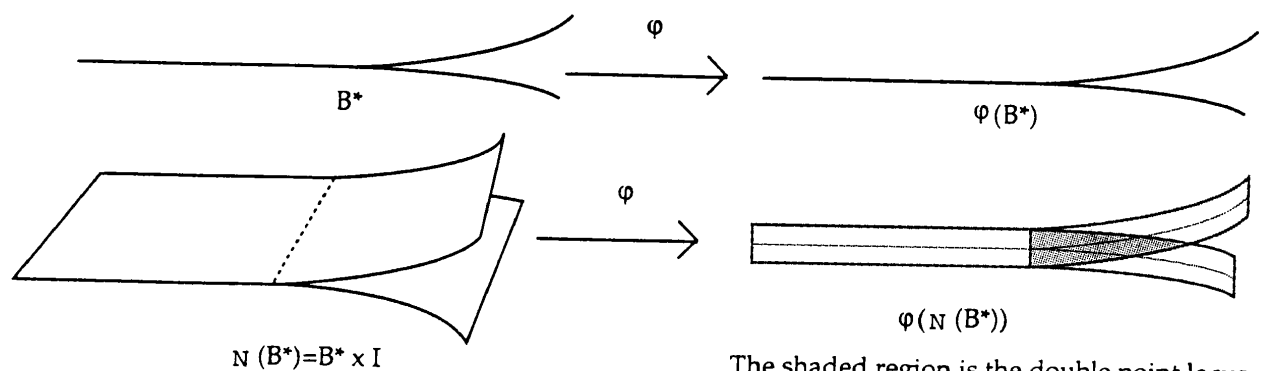

2-dimensional version of the normal neighborhood

Figure 1.2

We say that the branched surface $B_{1}$ is obtained from $B$ by splitting if there is 
a lift of $\phi_{1}: B_{1} \rightarrow M$ to an immersion $B_{1}^{*}$ to $\mathcal{N}\left(B^{*}\right)$ such that $B_{1}^{*}$ is transverse to the $I$-fibres, $B_{1}^{*}$ intersects each $I$-factor nontrivally, and finally $\partial B_{1}^{*} \subset \pi^{-1}\left(\partial B^{*}\right)$. Conversely we say that $B$ is obtained from $B_{1}$ by squeezing.

Remark 1.2. Figure 1.3a shows a splitting one dimension lower. Figure 1.3b describes a splitting in a 3-manifolds. Note that one crossed maw is created in Figure 1.3b.
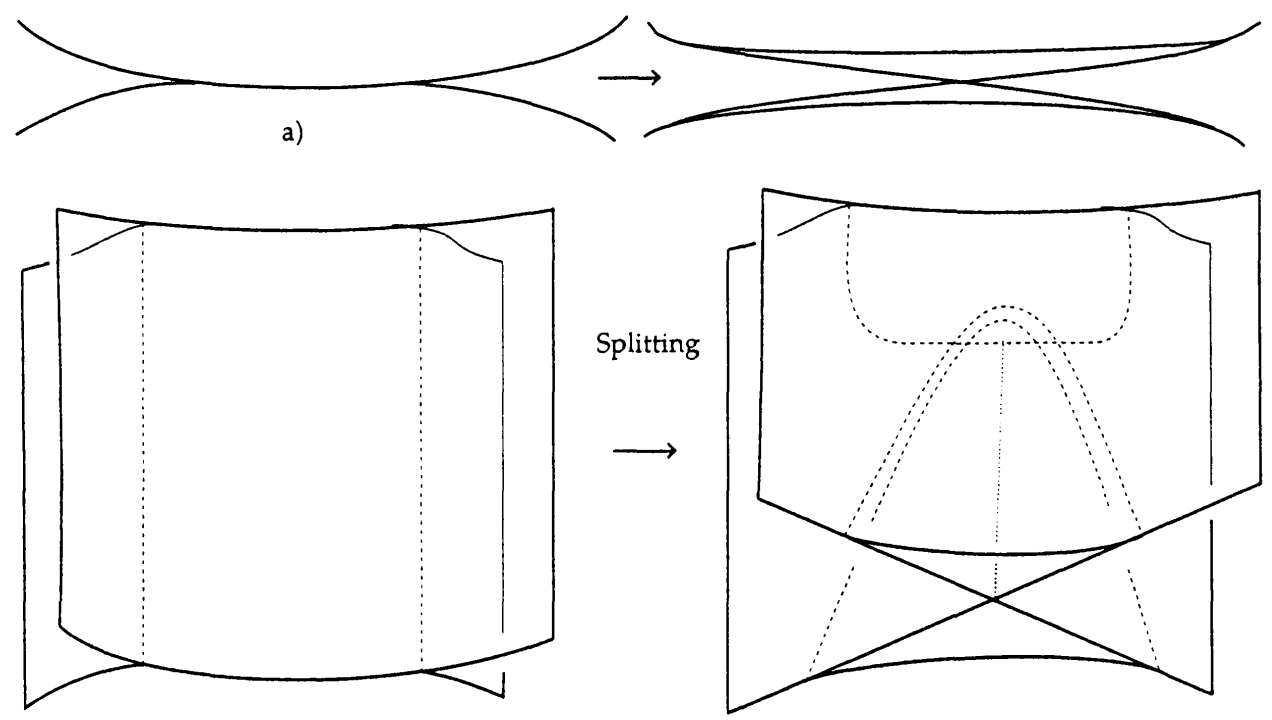

b)

Figure 1.3

Lemma 1.3. If $B_{1}$ is obtained from $B$ by splitting, then $B_{1}$ has a normal neighborhood $\mathcal{N}\left(B_{1}\right)$ which lifts to a singular immersion $\mathcal{N}\left(B_{1}^{*}\right) \subset \mathcal{N}\left(B^{*}\right)$ such that each I-fibre of $\mathcal{N}\left(B_{1}^{*}\right)$ is properly contained in the associated I-fibre of $\mathcal{N}\left(B^{*}\right)$.

Definition 1.4. Suppose that we are given a Riemannian metric on $B$. If $C>0$, then we define a $C$-splitting to be a splitting $B \rightarrow B_{1}$ such that if $i: \mathcal{N}\left(B_{1}^{*}\right) \rightarrow$ $\mathcal{N}\left(B^{*}\right)$ is the associated singular immersion then there does not exist paths $\alpha, \beta$ : $[0, t+\epsilon] \rightarrow \mathcal{N}\left(B_{1}^{*}\right)$ such that $\alpha(0), \beta(0)$ lie in the same $I$-fibre of $\mathcal{N}\left(B_{1}^{*}\right)$, for all $s \in$ $[0, t], i(\alpha(s)), i(\beta(s))$ lie in the same $I$-fibre of $\mathcal{N}\left(B^{*}\right)$, and for $s>t ; i(\alpha(s)), i(\beta(s))$ 
lie in distinct $I$-fibres of $\mathcal{N}\left(B^{*}\right)$. Finally length $\pi(i(\alpha([0, t])))<C$. Intuitively this means that the branched locus of $B$ has been blasted open at least distance $C$.

Definition 1.5. By a 2-dimensional abstract lamination $\mu$ we mean a topological space covered by charts of the form $U_{i}=T_{i} \times \mathbb{R}^{2}$, where $T_{i}$ is a closed subset of $[0,1]$. Furthermore if $U_{i} \cap U_{j} \neq \emptyset$, then the coordinate transformations are of the form $(x, y) \rightarrow(\psi(x), \phi(x, y))$ where $x \in T_{i}, y \in \mathbb{R}^{2}$. This is a specialization of a more general definition given by [Ca]. There Alberto Candel initiates a deep investigation of the differential geometry of 2-dimensional abstract laminations.

Throughout this paper we will refer to abstract laminations simply as laminations. We say that the lamination $\mu$ is immersed in $M^{3}$ if there is a continuous map $J: \mu \rightarrow M^{3}$ whose restriction to each leaf of $\mu$ is a smooth or PL immersion. We say that $\mu$ is carried by the branched surface $B$ if the mapping $J$ factors through an immersion into $\mathcal{N}\left(B^{*}\right)$ such that the leaves of $\mu$ are transverse to $I$-fibres of $\mathcal{N}\left(B^{*}\right)$. If $\mu$ intersects each $I$-fibre of $\mathcal{N}\left(B^{*}\right)$, then $\mu$ is fully carried by $B$.

Definition 1.6. If $\lambda$ is carried by $B$, then $B_{1}$ is said to be a $\lambda$-splitting of $B$ if there exists a lift of $\lambda$ to $\mathcal{N}\left(B^{*}\right)$ which after normal homotopy lifts to $\mathcal{N}\left(B_{1}^{*}\right)$.

Definition 1.7. Generalizing the similar notion of [GO] we say that an infinite sequence of branched surfaces $B_{1}, B_{2}, \cdots$ is a full splitting if there exists $C>0$ such that for each $i$ there exists a $j(i)$ such that the splitting $B_{i} \rightarrow B_{j(i)}$ is a $C$-splitting. If $B$ is the first term of a full splitting, then we say that $B$ is fully splittable.

The following result is the direct analogue of Lemma 4.3 [GO] to immersed laminations. See $[\mathrm{MO}]$ for a version for laminations in finite 2-complexes.

Theorem 1.8. $B$ is fully splittable if and only if $B$ fully carries a lamination.

Proof. Suppose that $B$ is fully splittable. After making the lengths of the $I$-fibres of $\mathcal{N}\left(B_{i}\right)$ go to 0 as $i \rightarrow \infty$, then the inverse limit of the $B_{i}$ 's is a lamination fully carried by $B$. Conversely if $B$ fully carries $\lambda$, then $\lambda$ provides the clue to constructing a full splitting. The resulting lamination $\lambda^{\prime}$ arising from the inverse 
limit construction will generally be distinct from $\lambda$, however it will be normally homotopic to a sublamination of $\lambda$ in $\mathcal{N}\left(B^{*}\right)$.

Definition 1.9. Let $M$ be a triangulated 3-manifold, with triangulation $\tau$. Let $\tau^{i}$ denote its i-skeleton. Following Haken [Ha], if $J$ is a 1-manifold, then we say that $f: J \rightarrow \tau^{2}$ is normal if $f$ is a PL-immersion transverse to $\tau^{1}$ and for each edge $e$ of the induced triangulation on $J, f \mid e$ is an embedding which sends the vertices of $e$ to distinct 1-simplices of $\tau^{1}$. A normal 2-disc is a properly embedded 2-cell $D$ in a 3 -simplex $\sigma$ such that $\partial D$ is a normal curve crossing a given 1-simplex of $\sigma$ at most once. If $S$ is a surface, then we say $f: S \rightarrow M$ is normal or $S$ is a normal surface if $f$ is a branched immersion transverse to $\tau^{2}$ and each 2-cell $\eta$ in the induced cellulation $\kappa$ on $S$ gets mapped to a normal disc. Thus, all branch points occur in $\tau^{1}$. For $v \in \kappa^{0}$ define $s(v)$, the spinning of $v$, to be the number of 2-cells of $\kappa$ which meet $v$. If $e$ is a 1-simplex of $\tau$, define $V(e)$, the valence of $e$, to be the number of 2-cells of $\tau$ which meet $e$. Also for $v \in \kappa^{0}$, define $V(f(v))$ to be $V(e)$ where $f(v) \in e$. Thus $s(v) / V(f(v))$ measures the local branching of $f$ at $v$. We say that $f$ has fake boundary branching if there exists $v \in \kappa^{0} \cap \partial S$, such that $f$ is an immersion at $v$, but $s(v) / V(f(v)) \geq 1$.

Define $S(D)=\sum_{a \in \kappa^{0} \cap \partial D} s(a)$ to be the total spinning of $D$. Define $A(D)=$ $S(D) /\left(\left|\kappa^{0} \cap \partial D\right|\right)$ to be the average spinning of $D$.

Proposition 1.10. Let $\tau$ be a triangulation of the 3-manifold $M$. There exists a branched surface $\mathcal{B}$, called the canonical normal branched surface, with the following property. If $\lambda$ is any immersed lamination in $M$ whose leaves are normal with respect to $\tau$, then after normal homotopy $\lambda$ is carried by $\mathcal{B}$. In particular the conclusion holds if $\lambda$ is a compact normal surface, possibly with boundary.

Proof. Figure 1.4 shows how to construct a canonical branched surface $B$ on a 3simplex $\sigma$. It is symmetric under the symmetries of $\sigma$ and carries the isotopy class of each normal disc in a unique way. Thus by putting this branched surface in each 3-simplex of $\tau$ one obtains the desired $\mathcal{B}$. To start with, the three distinct normal quadralaterals and the four distinct normal triangles in $\sigma$ can be normally 
isotoped to appear in $\sigma$ as follows. The quadralaterals pairwise intersect in arcs whose endpoints lie on edges of $\sigma$. Also if $\eta$ is a triangle then $\eta \cap(\cup$ quads $)=\partial \eta$. Thus the union of the quadralaterals and triangles is isomorphic to the cone on the 1-skeleton of an octahedron together with four faces of the octahedron which pairwise meet in points. (I.e. one color of a two coloring of the octahedron.) See Figure 1.4a. By squeezing the faces in the appropriate manner, we obtain the desired branched surface $B$. See Figure $1.4 \mathrm{~b}$.

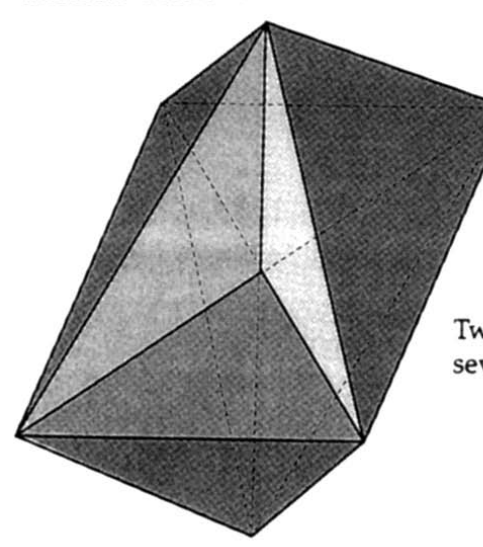

a)

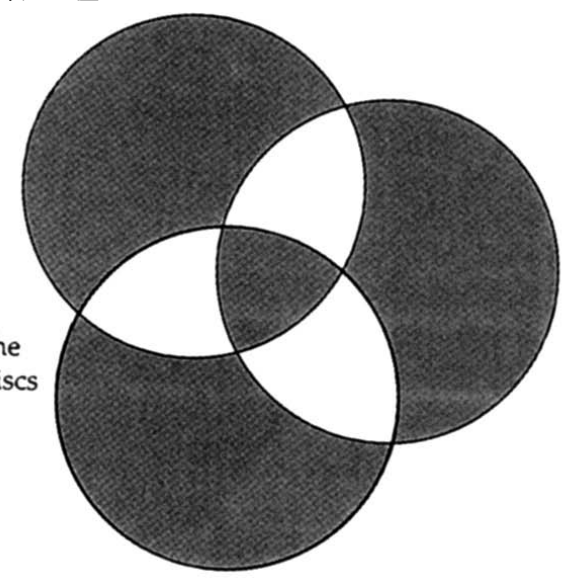

The shaded regions are the triangles. Attach hemispheres to each circle to obtain the quadralerals.
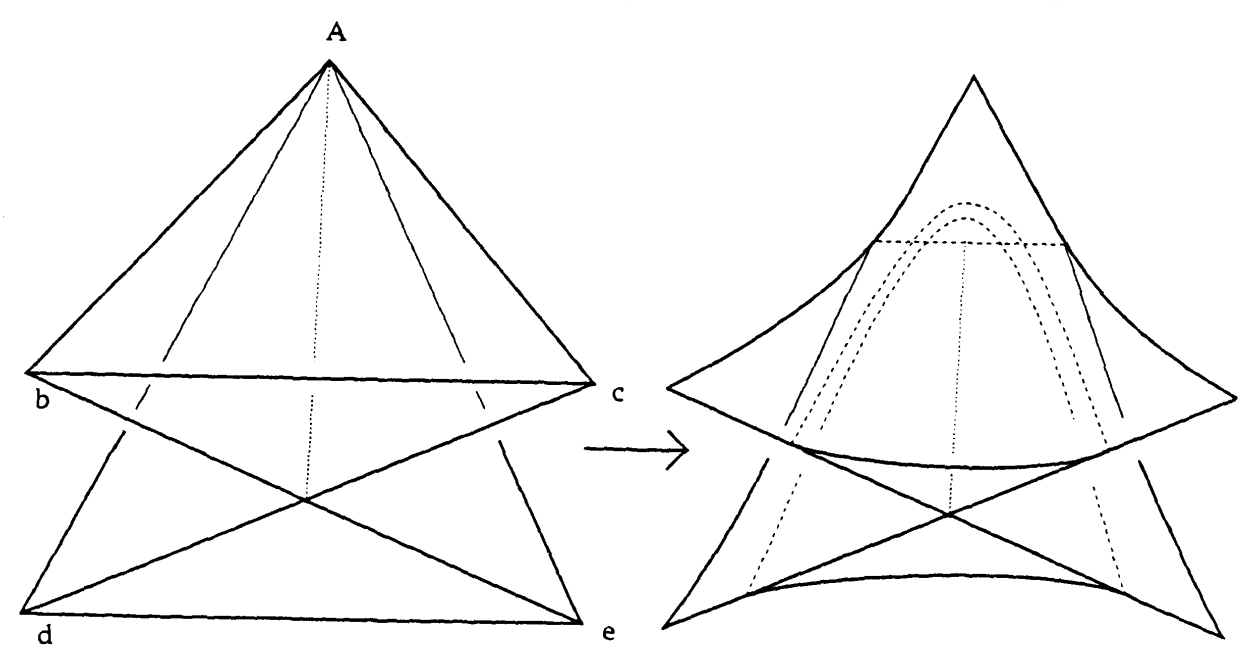

Attach discs to each of Abc, Ade, Abe, Adc.

Smoothing very near corner $A$ of the octahedron creates the branched surface $B$

b) 
Definition 1.11. A measured branched surface is a branched surface $B$ together with a non negative valued function $m:\{$ Sectors of $B\} \rightarrow \mathbb{R}$ such that if sectors $s_{1}, s_{2}$ merge into sector $s_{3}$, then $m\left(s_{1}\right)+m\left(s_{2}\right)=m\left(s_{3}\right)$. A measured lamination is a lamination $\lambda$ together with a transverse measure $m$. I.e. for each lamination chart $T_{i} \times \mathbb{R}^{2}$ there is a Borel measure supported on $T_{i}$. Furthermore the transversal overlap functions are measure preserving. If the measured lamination $(\lambda, m)$ is carried by the branched surface $B$, then $m$ induces a measure also called $m$ on $B$. Define the Euler characteristic of a measured branched surface as follows. If $(B, m)$ is a branched surface, then put a cell structure on $B$ so that the branched locus is contained in the 1-skeleton. If $t$ is a vertex or edge, define $m(t)$ to be the maximal $m$ value of a sector which contains $t$ and define $\chi(B, m)=\sum_{\text {vertices }} m\left(v_{i}\right)-\sum_{\text {edges }} m\left(e_{j}\right)+\sum_{f a c e s} m\left(f_{k}\right)$. This formula is invariant under splitting and choice of cell structure. If $(\lambda, m)$ is a measured lamination, carried by a branched surface $(B, m)$, then define $\chi(\lambda, m)=\chi(B, m)$.

Definition 1.12. We need the notion of diagram [LS] which is a slight generalization of a disc. (The first time reader is strongly advised to pretend that all diagrams are discs.) A diagram $D$ is a simply connected finite 2-complex that is embedded in the plane. Define $\stackrel{\circ}{D}$ to be the interior of $D$ as a subset of $\mathbb{R}^{2}$. Define $\partial(D)=D-\stackrel{\circ}{D}$ and $\operatorname{Bd}(D)$ to be a simplicial map of the circle to the curve which traverses around $D$. See Figure 1.5. Call a diagram map $f: D \rightarrow M$ admissible if $\partial D$ has a triangulation $\phi$ such that $f$ takes each 1-cell of $\phi$ to a normal arc and $f$ is transverse to $\tau^{2}$, except at isolated points where $f$ appears as in Figure 1.6. We say that $g: S^{1} \rightarrow \tau^{2}$ extends to the diagram $G: D \rightarrow M$ or $G$ spans $g$, if $G \circ \mathrm{Bd}(D)$ is normally homotopic to $g$. If $f: D \rightarrow M$ is an admissible diagram map, then define length( $\partial f)$ to be the number of 1-cells in the induced triangulation on $\operatorname{Bd}(D)$. 


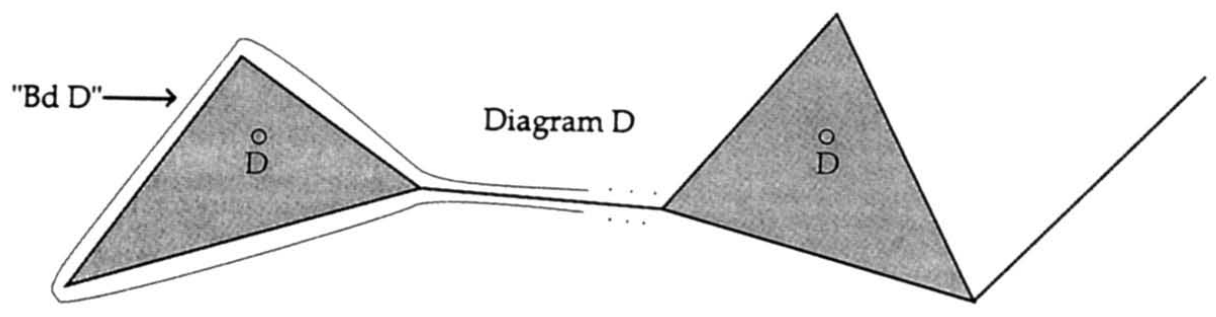

Figure 1.5

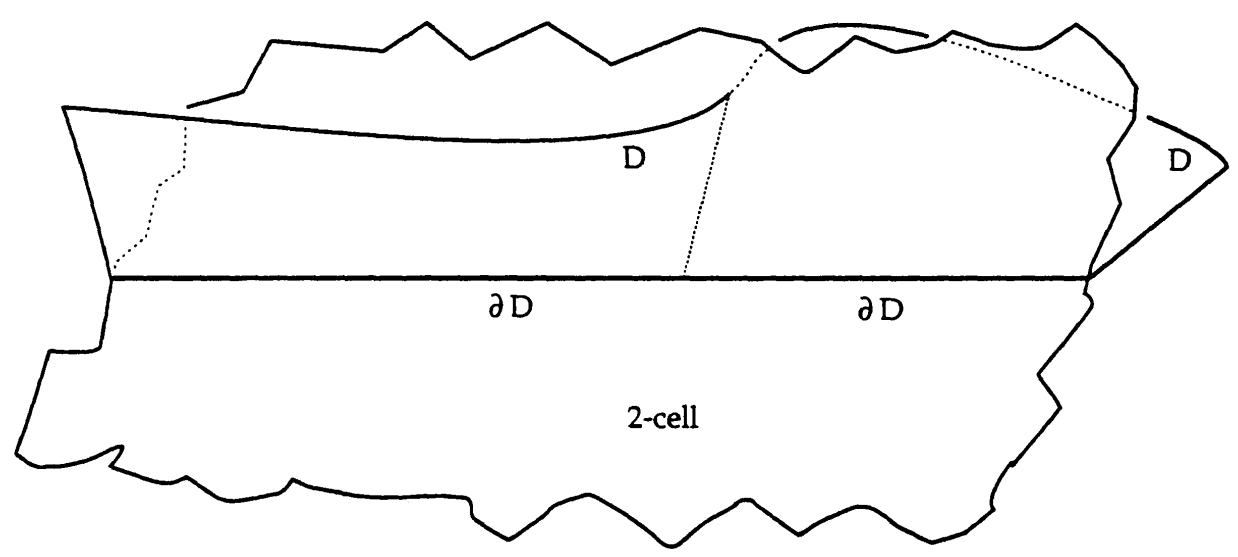

Figure 1.6

Definition 1.13. If $f: S \rightarrow M$ is a normal surface, then define "area (f)" $=\sum_{i=1}^{n} s\left(v_{i}\right) / V\left(f\left(v_{i}\right)\right)$, where $\left\{v_{1}, \cdots, v_{n}\right\}$ are the vertices in the induced cellulation $\kappa$ on $S$. Thus if $f: S \rightarrow M$ is a normal immersion of a closed surface, then $\operatorname{area}(f)$ is the number of vertices in $\kappa$, i.e. the Haken weight. This definition of 
area takes into account local branching and is additive under union. In a similar manner we can define area $(f)$ where $f: D \rightarrow M$ is an admissible diagram map, or if $f$ is a map of a compact surface such that $f \mid \partial D$ is normal and $f$ is transverse to $\tau^{2}$ except as in Figure 1.6. In these cases one uses the induced stratification on $D$ to compute $s\left(v_{i}\right)$ and sum over points of $f^{-1}\left(\tau^{1}\right)$.

A diagram $f: D \rightarrow M$, and in particular a normal map of a disc, is said to be least area (resp. $k$-quasi least area) if $\operatorname{area}(D) \leq \operatorname{area}(E)$ (resp. $\operatorname{area}(D) \leq k$ $\operatorname{area}(E))$ among all diagrams $E$ spanning $\partial D$.

A normal surface $f: L \rightarrow M$ is least area if the restriction of $f$ to embedded discs is a least area diagram. $L$ is strongly least area $[\mathrm{MO}]$ if the induced immersion of the universal covering of $L$ into $M$ is least area. In a similar manner define strongly $k$-quasi least area for a normal map $f: L \rightarrow M$. The leaves of a lamination are quasi strongly least area if there exists a $k$ such that for each leaf $L$, the induced immersion of $L$ into $M$ is strongly $k$-quasi least area.

The following Proposition is a restatement of the main technical result of Mosher and Oertel [MO]. We translate their work about PL maps of discs into finite 2complexes into a Proposition about maps of discs into 3-manifolds. We generalize slightly to allow for $k$-quasi least area maps.

Proposition 1.14 [MO]. Let $D_{1}, D_{2}, \cdots$ be a sequence of immersed normal discs, in the triangulated compact 3-manifold $M$, such that $\lim _{i \rightarrow \infty}$ length $\left(\partial D_{i}\right) /$ area $\left(D_{i}\right) \rightarrow 0$. If no $D_{i}$ has fake boundary branching, then after passing to a subsequence, $D_{i}$ converges to a normal Euler characteristic-0 measured lamination $\lambda$. If each of the discs is $k$-quasi least area, then each leaf of $\lambda$ is strongly $k$-quasi least area. If each $D_{i}$ is least area, then $\lambda$ has no 2-sphere leaves. Finally, the induced metric on each leaf is complete.

Remarks. The proof of the existence of $\lambda$ in [MO] is greatly complicated by the fact that it is happening in the setting of branched immersed discs into 2-complexes. In the setting of immersed normal discs without fake boundary branching in 3manifolds the argument is considerably simpler. Mosher and Oertel do a competent 
job of explaining their proof of strong area minimization. Indeed, their argument shows that if $\lambda$ had a 2-sphere leaf, then for $i$ sufficiently large, some component of $D_{i}$ would be a 2 -sphere, which gives a contradiction. By construction each leaf of $\lambda$ is complete. One should be aware that the discussion of diagrams is implicit, though suppressed in [MO].

Idea of the proof of existence of $\lambda$. Each $D_{i}$ is carried by $\mathcal{B}$, the canonical normal branched surface. Fix a small $C>0$. By the usual diagonal argument one passes to a subsequence of the $D_{i}$ 's and finds a sequence $\mathcal{B}=B_{0}, B_{1}, \cdots$ such that $B_{i}$ is obtained from $B_{i-1}$ via $C$-splitting where each $B_{j}$ carries every $D_{i}$ for $i \geq j$. (This step would fail if there was uncontrolled fake boundary branching.) By counting the number of times a given $D_{i}$ crosses a sector of $B_{j}$, we obtain a function $m_{i j}$ on the sectors of $B_{j}$ which fails to be a transverse measure only because of the boundary of $D_{i}$. Let $a_{i j}$ be the maximal value of $m_{i j}$. By considering $m_{i j} / a_{i j}$ and using the length area hypothesis, then by passing to a subsequence of the $D_{i}$ 's the measures $m_{i 1} / a_{i 1}$ converge to a fixed transverse measure $m_{1}$ on $B_{1}$. By passing to another subsequence we can assume that the $D_{i}$ 's induce a measure $m_{2}$ on $B_{2}$ so that as measured laminations $\left(B_{2}, m_{2}\right)$ is obtained by splitting $\left(B_{1}, m_{1}\right)$. Thus by applying Theorem 1.8 to the sequence $\left\{\left(B_{i}, m_{i}\right)\right\}$ we obtain a measured lamination $\lambda^{\prime}$. Finally take $\lambda$ to be the sublamination which is the support of the measure $m$. This type of argument, in tamer form, goes back to Plante [Pl].

Since $\chi\left(D_{i}\right)=1$, the Euler characteristic of the approximate measure $m_{i j} / a_{i j}$ on $B_{j}$ is approximately $1 / a_{i j}$ and $m_{i j} / a_{i j} \rightarrow m_{j}$, it follows that the Euler characteristic of $\lambda$ is zero.

Definition 1.15. Let $\lambda$ be a lamination with a piecewise Riemannian metric on each leaf, which varies continuously in the transverse direction. (E.g. a metric induced from a 3-manifold) We say $\lambda$ is conformally Euclidean if each leaf is conformally equivalent to the Euclidean plane. A measured Riemannian lamination $\lambda$ is said to be semi-Euclidean if the set of leaves conformally equivalent to the Euclidean plane are of full measure and dense in $\lambda$. 
The following result provides the crucial link between the topology of the measured laminations and their geometry.

Theorem (Ghys [Gh] 1.16). Let $\lambda$ be a measured 2-dimensional abstract Riemannian lamination with out spherical leaves. $\chi(\lambda)=0$ if and only if almost all leaves are conformally Euclidean.

Remark 1.17. This was proven originally for 2-dimensional foliations of manifolds, but Candel $[\mathrm{Ca}]$ pointed out that the proof holds for 2-dimensional abstract Riemannian laminations.

\section{$\S 2$ \\ IMMERSED LEAST AREA \\ Semi-EuClidean Laminations}

Theorem 2.1. If $M$ is a closed non negatively curved aspherical 3-manifold, then there exists an immersed strongly least area, Euler characteristic 0, measured semiEuclidean lamination $\lambda$. The induced metric on each leaf is complete.

Remark 2.2. i) Here $M$ has a triangulation $\tau$, and the various measurements of length and area are computed simplically.

ii) Theorem 2.1 should be viewed as a refinement of [MO]. Their work translated to closed 3-manifolds yields the above theorem with immersed replace by branched immersed.

Conjecture 2.3. If $K_{0}$ is a non negatively curved 2-complex, then $K_{0}$ is simple homotopy equivalent to a 2-complex $K_{1}$ such that $K_{1}$ supports an immersed least area Euler characteristic zero semi-Euclidean lamination.

The following is the main result of this section.

Proposition 2.4. Let $M$ be a closed 3-manifold with triangulation $\tau . \pi_{1}(M)$ is not negatively curved if and only if there exists a sequence of least area normal immersed discs $f_{i}: D_{i} \rightarrow M$, such that length $\left(\partial f_{i}\right) /$ area $\left(f_{i}\right) \rightarrow 0$ and no $f_{i}$ has fake boundary branching. 
Remark. This result is a serious technical advance beyond the obvious translation of Gromov's theorem, group negative curvature implies linear isoperimetric inequality, to normal surfaces in 3-manifolds. The new wrinkle is that "no $f_{i}$ has fake boundary branching."

\section{Proof of Proposition 2.4.}

Step 0. $\pi_{1}(M)$ is non negatively curved if and only if there exists a sequence of least area normal immersed discs $f_{i}: D^{2} \rightarrow M$, such that length $\left(\partial f_{i}\right) / \operatorname{area}\left(f_{i}\right) \rightarrow 0$.

Proof of Step 0. Since $\pi_{1}\left(\tau^{2}\right)=\pi_{1}(M)$ is not negatively curved, there exists by Gromov [Gr], [Bo] a sequence of simplicial maps of discs $a_{i}: A_{i} \rightarrow \tau^{2}$ such that $\operatorname{length}_{\tau}\left(A_{i}\right) / \operatorname{area}_{\tau}\left(A_{i}\right) \rightarrow 0$. Where length $\left(\partial A_{i}\right)$ (resp. area ${ }_{\tau}\left(A_{i}\right)$ is the number of 1-simplices (resp. 2-simplices) in $\partial A_{i}$ (resp. in $A_{i}$.) Furthermore area $a_{\tau}\left(A_{i}\right)$ is minimal among all such simplicial maps of diagrams that span $a_{1} \mid \partial A_{i}$.

Its routine to translate this statement about 2-complexes to the statement of Step 0.

From now on we will assume that $\pi_{1}(M)$ is non negatively curved and that Proposition 2.4 is false. Thus there exists a constant $K_{0} \leq 1$ such that if $f$ : $D \rightarrow M$ is least area normal immersed disc with no fake boundary branching, then $\operatorname{length}(\partial f) / \operatorname{area}(f)>K_{0}$.

Here is how we derive a contradiction. As a result of Steps 0 and 1 we find a sequence satisfying the conclusion of Proposition 2.4, except that each disc has fake boundary branching at a single vertex. We then delete a small neighborhood of the branching vertex and take (in Step 2) a weak limit of these trimmed discs to obtain an immersed least area measured lamination $\lambda^{\prime}$ of Euler characteristic 0 , with boundary $S^{1} \times K, K$ a closed subset of $[0,1]$. By capping off the boundary circles with discs, we obtain a measured lamination $\lambda$ of positive Euler characteristic and derive a contradiction to the least area property of $\lambda$.

Step 1. There exists a sequence of least area normal immersed $\operatorname{discs} f_{i}: D^{2} \rightarrow M$, such that length $\left(\partial f_{i}\right) / \operatorname{area}\left(f_{i}\right) \rightarrow 0$. Furthermore all the fake boundary branching 
of $f_{i}$ occurs at a single vertex $w_{i}$ of $\partial D_{i}$.

Proof of Step 1. Let $f_{i}$ be as in Step 0 and let $\Delta_{i}$ denote the cellulation induced on $D_{i}$ by $\tau$. If $J$ is a subcomplex of a cell complex, then let $\operatorname{St}(J)$ denote the union of all open cells whose closures intersect $J$ and $\bar{S} t(J)$ to denote the closure of $\operatorname{St}(J)$. Define $D_{i}^{\text {trim }}=D_{i}-S t\left(\partial D_{i}\right)$.

Claim 0. We can assume that $D_{i}^{\text {trim }}$ is connected, that $\Delta_{i}$ has no separating 1-cells, and $\bar{S} t(v) \cap D_{i}^{t r i m}$ is nonempty and connected for each vertex $v \in \partial D_{i}$.

Proof of Claim 0. Consider a maximal collection of properly embedded $\operatorname{arcs} \delta_{j}$ in $D_{i}$ such that $\partial \delta_{j} \subset \Delta^{0}$, interiors of the $\delta_{j}$ 's are pairwise disjoint and the $\delta_{j}$ 's satisfy the following additional property. Either $\delta_{j}$ is a separating 1-cell of $\Delta_{i}$, or $\stackrel{\circ}{\delta}_{j} \subset \Delta_{i}-\Delta_{i}^{2}$ and each component of $D_{i}-\delta_{j}$ contains at least one open 2 cell of $\Delta_{i}$. The number of such paths between two given vertices is uniformly bounded by a constant $K_{1}$, since $M$ is compact and $D_{i}$ is least area. Thus for Euler characteristic reasons there are at most $K_{1}$ length $\left(\partial D_{i}\right)$, such $\delta_{j}$ 's. If $C_{n}^{\prime}$ is a component of $D_{i}-\cup \delta_{j}$, then let $C_{n}$ be the smallest subcomplex of $\Delta_{i}$ which contains $C_{n}^{\prime}$. Since $\sum_{n}$ length $\left(\partial C_{n}\right) \leq 4 K_{1}\left(\right.$ length $\left.\left(\partial D_{i}\right)\right)$, some $C_{n}$ will have very small isoperimetric ratio, if $D_{i}$ does. Finally $C_{n}$ satisfies the conclusions of Claim 0 . Thus by cutting down the $D_{i}$ 's we can assume that they satisfy the conclusions of both Step 0 and Claim 0.

We will supress cumbersome language by writing as if each $D_{i}^{\text {trim }}$, as well as other to be defined objects are discs rather than diagrams.

If $v \in \Delta^{0} \cap \partial D_{i}$, then define $D_{i}^{v}=D_{i}^{t r i m} \cup \bar{S} t(v)$. $D_{i}^{v}$ is an immersed disc with possible fake boundary branching only at $v_{i}$. Since $D_{i}^{\text {trim }}$ is an immersed least area disc with no fake boundary branching, it follows that for all $i$, length $\left(\partial D_{i}^{\text {trim }}\right) / \operatorname{area}\left(D_{i}^{\text {trim }}\right)>K_{0}$.

Claim 1. The average spinning $A\left(D_{i}\right) \rightarrow \infty$.

Proof. Otherwise $A\left(D_{i}\right) \leq A$ all $i$ implies length $\left(\partial D_{i}^{\text {trim }}\right) / \operatorname{area}\left(D_{i}^{\text {trim }}\right) \leq$ $2 S\left(D_{i}\right) /\left(\operatorname{area}\left(D_{i}\right)-S\left(D_{i}\right)\right) \leq 2 A \operatorname{length}\left(\partial D_{i}\right) /\left(\operatorname{area}\left(D_{i}\right)-A \operatorname{length}\left(\partial D_{i}\right)\right) \rightarrow 0$ as 
$i \rightarrow \infty$.

We now define $\operatorname{Fan}_{j}(v)$, which more or less is the smallest simply connected subset of $D_{i}^{v}(v)$ which contains all simplices distance $j$ from $v$. More precisely, if $v$ is a vertex of $\partial D_{i}$ then define inductively define $S t_{n}^{\text {trim }}(v)=\bar{S} t\left(S t_{n-1}^{t r i m}(v)\right)$ where $S t_{1}^{\text {trim }}(v)=\bar{S} t(v)$, all these complexes being computed in $D_{i}^{v}$. Let $\operatorname{Fan}_{j}(v)$ be the minimal simply connected subcomplex of $D_{i}^{v}$ which contains $S t_{j}^{t r i m}(v)$.

Claim 2. Given $p$, there exists $B(p)$ independent of $i$, such that for any $v_{r}, v_{s} \in \partial D_{i}$, $\operatorname{area}\left(S t_{p}^{\text {trim }}\left(v_{r}\right) \cap S t_{p}^{\text {trim }}\left(v_{s}\right)\right)<B(p)$. (Recall $S t_{p}^{\text {trim }}\left(v_{r}\right) \subset D_{i}^{v_{r}}$ and $S t_{p}^{\text {trim }}\left(v_{s}\right) \subset$ $D_{i}^{v_{s}}$.)

Proof. If $x$ is a vertex in $S t_{p}^{\text {trim }}\left(v_{r}\right) \cap S t_{p}^{t r i m}\left(v_{s}\right)$, then there exists a simplicial path $\alpha_{x}$ of length $\leq 4 p$ from $v_{r}$ to $v_{s}$ which passes through $x$. The union of two such paths $\alpha_{x}, \alpha_{y}$ gives rise to a least area diagram $D_{x y} \subset D_{i}$, with $\partial D_{x y} \subset \alpha_{x} \cup \alpha_{y} \subset D_{x y}$ and hence length $\partial D_{x y} \leq 8 p$. Since $M$ is compact, the number of such diagrams is bounded and hence if $x, y \in S t_{p}^{\text {trim }}\left(v_{r}\right) \cap S t_{p}^{\text {trim }}\left(v_{s}\right)$, then the distance between $x$ and $y$ in $D_{i}^{\text {trim }}$ is uniformly bounded, independent of $i$. Since the valence of vertices of $D_{i}^{\text {trim }}$ are uniformly bounded, independent of $i$, Claim 2 follows.

Claim 3. Given $p$, there exists a constant $K_{3}$ independent of $i$ such that at most $K_{3}\left(\right.$ length $\left(\partial D_{i}\right)$ distinct pairs $\left(\operatorname{Fan}_{p}\left(v_{r}\right), \operatorname{Fan}_{p}\left(v_{s}\right)\right)$ intersect.

Proof. If $\operatorname{Fan}_{p}\left(v_{r}\right) \cap \operatorname{Fan}_{p}\left(v_{s}\right) \neq \emptyset$, then define $\alpha_{r s}$ to be a shortest simplicial path in 1-cells of $D_{i}^{v_{r}} \cup D_{i}^{v_{s}}$ from $v_{r}$ to $v_{s}$. Thus $\alpha_{r s}$ is embedded of length $\leq 4 p$. By rechoosing $\alpha_{r s}$ we can assume that $D_{i}^{\text {trim }} \cap \alpha_{r s}$ is connected, and using Claim 0 that either $\alpha_{r s} \cap D_{i}^{\text {trim }} \neq \emptyset$ or $\alpha_{r s} \subset \partial D_{i}$. Furthermore, if $\alpha_{r s} \cap D_{i}^{\text {trim }} \neq \emptyset$, then after possibly deleting the first and/or last 1-cells of $\alpha_{r s}$, the remainder is properly embedded in $D_{i}$. Also if $\alpha_{r s} \subset \partial D_{i}$, then length $\left(\alpha_{r s}\right) \leq 4$. Thus there are at most 4 length $\left(\partial D_{i}\right)$ peripheral $\alpha_{r s}$ 's and at most $5 N$ non peripheral $\alpha_{r s}$ 's, where $N$ is the number of properly embedded $\alpha_{r s}$ 's. Since at most finitely many paths $\alpha_{r s}$ can pass through a given vertex of $D_{i}^{t r i m}$, it follows that there exists a uniform bound $K_{2}$ on the number of $\alpha_{r s}$ 's which can intersect a given $\alpha_{u v}$ inside of $D_{i}^{t r i m}$. The collection 
of properly embedded $\alpha_{r s}$ 's can be partitioned into $K_{2}+1$ subsets, with the property that if $\alpha_{r s}, \alpha_{u v}$ are distinct elements of a given subset, then $\stackrel{\circ}{\alpha}_{r s} \cap \stackrel{\circ}{\alpha}_{u v}=\emptyset$. For Euler characteristic reasons each subset has at most length $\left(\partial D_{i}\right)-1$ elements, so $N \leq\left(K_{2}+1\right)\left(\right.$ length $\left.\left(\partial D_{i}\right)-1\right)$ and Claim 3 follows with $K_{3}=5\left(K_{2}+1\right)+4$.

Claim 4. For every $k>0$, there is a $N>0$ such that $i>N$ implies that there

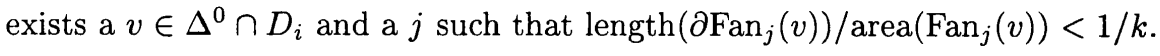

Proof. Fix k and i. If $\mathrm{v}$ is a vertex in $\partial D_{i}$, then let $\partial_{e x t} \operatorname{Fan}_{j}(v)$ denote $\partial \operatorname{Fan}_{j}(v) \cap$ $\stackrel{\circ}{D}_{i}^{v}$. If there exists $j$ such that $\operatorname{length}\left(\partial_{e x t} \operatorname{Fan}_{j}(v)\right) \leq s(v) / 2 k$, then length $\left(\partial \operatorname{Fan}_{j}(v)\right) / \operatorname{area}\left(\operatorname{Fan}_{j}(v)\right) \leq\left(s(v) / 2 k+\operatorname{length}\left(\operatorname{Fan}_{j}(v) \cap \partial\left(D_{i}^{v}\right)\right)\right) / s(v)$ and hence either length $\left(\partial \operatorname{Fan}_{j}(v)\right) / \operatorname{area}\left(\operatorname{Fan}_{j}(v)\right)<1 / k$ would hold or length $\left(S t_{j}^{\text {trim }}(v) \cap \partial D_{i}^{v}\right)=$ length $\left(\operatorname{Fan}_{j}(v) \cap \partial D_{i}^{v}\right) \geq s(v) / 2 k$. In the latter case, for all $p \geq j$, length $\left(S t_{p}^{\text {trim }}(v) \cap \partial D_{i}^{v}\right) \geq s(v) / 2 k$.

Now choose $p$ so that $p /(6 k) \geq 8 / K_{0}$. Therefore for all vertices $v$ of $\partial D_{i}$

i) length $\left(\partial \operatorname{Fan}_{j}(v)\right) / \operatorname{area}\left(\operatorname{Fan}_{j}(v)\right)<1 / k$ for some $j \leq p$ or

ii) length $\left(S t_{p}^{\text {trim }}(v) \cap \partial D_{i}^{v}\right) \geq s(v) / 2 k$ or

iii) $\operatorname{area}\left(S t_{p}^{\text {trim }}(v)\right) \geq 8 s(v) / K_{0}$.

Indeed if neither i) nor ii) held for $v$, then for all $j \leq p$, length $\left(\partial_{e x t} \operatorname{Fan}_{j}(v)\right) \geq$ $s(v) / 2 k$ and hence $\operatorname{area}\left(S t_{p}^{t r i m}(v)\right) \geq(1 / 3) \sum_{j=1}^{p} \operatorname{length}\left(\partial_{e x t} \operatorname{Fan}_{j}(v)\right) \geq p s(v) / 6 k \geq$ $8 s(v) / K_{0}$. This argument was inspired by $[\mathrm{Pa}]$.

Let $v_{1}, \cdots, v_{m}$ denote the vertices of $\partial D_{i}$. It follows that either

a) length $\left(\partial \operatorname{Fan}_{j}\left(v_{s}\right)\right) / \operatorname{area}\left(\operatorname{Fan}_{j}\left(v_{s}\right)\right)<1 / k$ for some vertex $v_{s}$ of $\partial D_{i}$ and some $j \leq p$ or

b) $\sum_{n=1}^{m} \operatorname{length}\left(S t_{p}^{\text {trim }}\left(v_{n}\right) \cap \partial D_{i}^{v}\right) \geq S\left(D_{i}\right) / 4 k$ or

c) $\sum_{n=1}^{m} \operatorname{area}\left(S t_{p}^{t r i m}\left(v_{n}\right)\right) \geq 4 S\left(D_{i}\right) / K_{0}$.

We show that b) does not hold if $i$ is sufficiently large. Claims 2,3 imply that the sum of the pairwise area overlaps of the $S t_{p}^{t r i m}$ 's is bounded above by $B(p) K_{3}$ length $\left(\partial D_{i}\right)$. Possibility b) implies that the sum of the total pairwise area overlaps of the $S t_{p}^{\text {trim }}$ 's is $\geq S\left(D_{i}\right) / 12 k=A\left(D_{i}\right) \operatorname{length}\left(\partial D_{i}\right) / 12 k$. Now apply Claim 1. 
We show that $\mathrm{c}$ ) does not hold if $i$ is sufficiently large. If $c$ ) holds then we $\operatorname{obtain} \operatorname{area}\left(D_{i}^{\text {trim }}\right) \geq \operatorname{area}\left(\left(\cup_{n=1}^{m} S t_{p}^{\text {trim }}\left(v_{n}\right)\right)-\operatorname{St}\left(\partial D_{i}\right)\right) \geq 4 S\left(D_{i}\right) / K_{0}-B(p) K_{3}$ $\operatorname{length}\left(\partial D_{i}\right)-S\left(D_{i}\right) \geq 2 S\left(D_{i}\right) / K_{0}+S\left(D_{i}\right)-B(p) K_{3} \operatorname{length}\left(\partial D_{i}\right) \geq$ length $\left(\partial D_{i}^{\text {trim }}\right) / K_{0}+\left(A\left(D_{i}\right)-B(p) K_{3}\right)$ length $\left(\partial D_{i}\right)>\operatorname{area}\left(D_{i}^{\text {trim }}\right)$. By Claim 1 , the latter inequality holds if $i$ is sufficiently large.

Thus for fixed $k$, a) holds for $i$ sufficienlty large.

Thus we can assume that each $D_{i}$ has fake branching except at the vertex $w_{i}$. Furthermore by the compactness of $M$ we can assume that for all $i, f\left(w_{i}\right)=w \in \tau^{1}$. Let $E_{i}=D_{i}-\stackrel{\circ}{S} t\left(v_{i}\right)$ and $h_{i}=f_{i} \mid\left(D_{i}-\stackrel{\circ}{S} t\left(w_{i}\right)\right)$. Let $\gamma$ be the 1-simplex of $\tau$ which contains $w$ and let $\alpha_{i}=\partial \operatorname{St}\left(w_{i}\right)-\partial D_{i}$. Since $h_{i}$ has no branching or fake boundary branching we can assume that for all $i$, length $\left(\partial h_{i}\right) / \operatorname{area}\left(h_{i}\right)>K_{0}$. Thus there exists $K_{7}>0$ so that for $i$ sufficiently large length $\left(\alpha_{i}\right) / \operatorname{area}\left(E_{i}\right)>K_{7}$ which in particular implies that length $\left(\partial E_{i}-\alpha_{i}\right) /$ length $\left(\alpha_{i}\right) \rightarrow 0$. Since the restriction of $f_{i}$ to $S t\left(w_{i}\right)$ is an immersion, $h_{i}\left(\alpha_{i}\right)$ is an embedded arc which spirals around $\partial \bar{S} t(\gamma)$.

Step 2. Either Proposition 2.4 is true or there exists an immersed normal measured lamination $(\lambda, m)$ by strongly least area leaves such that $\chi(\lambda)=0$. Furthermore $\partial \lambda$ is a lamination of the form $S^{1} \times K \subset \partial \bar{S} t(\gamma)$ where $K$ is compact and $m(K)=1$.

Proof of Step 2. Let $n_{i}$ be the number of times $h\left(\alpha_{i}\right)$ spirals around $\operatorname{St}(\gamma)$. Here we abuse notation by letting $\operatorname{St}(\gamma)$ denote the union of all 3-simplices of $\tau$ which nontrivially intersect $\stackrel{\circ}{\gamma}$. As in the proof of Proposition 1.14 normalize the various weights associated to the discs $E_{i}$ by dividing by $n_{i}$. Since area $\left(D_{i}\right) / n_{i}$ is uniformly bounded, thus we can apply the proof of Proposition 1.14 to let the normalized discs $E_{i} / n_{i}$ weakly converges to the measured lamination $(\lambda, m)$. Since $\partial \lambda$ is the weak limit of the sequence $\partial E_{i} / n_{i}$ and length $\left(\partial E_{i}-\alpha_{i}\right) / n_{i}=0$, any weak limit of $\partial E_{i} / n_{i}$ is a weak limit of $h_{i}\left(\alpha_{i}\right) / n_{i}$. Because each $D_{i}$ is immersed in $M, \alpha_{i}$ is an embedded spiral in $\partial \operatorname{St}(\gamma)$, so it follows that any weak limit is of the form $S^{1} \times K$ where $K$ is compact and $m(K)=1$. 
Step 3. Obtain the contradiction.

Proof of Step 3. Let $\left(\lambda^{\prime}, m^{\prime}\right)$ be the immersed measured lamination obtained from $(\lambda, m)$ by capping off each component of $S^{1} \times K$ by a disc. $\chi\left(\lambda^{\prime}\right)=1$ and so by the Connes sphere theorem [Co] (or [MO1] for an elementary proof) there exists a measured sublamination $\mathcal{S}$ of $\lambda^{\prime}$ consisting only of 2 -spheres and $\chi(\mathcal{S}) \geq 1$.

Since $\lambda$ is strongly area minimizing it has no 2 -sphere leaves. The rest of the argument follows implicitly from the proof of "strong area minimization of leaves theorem" [MO]. See the next paragraph for a hint. If some leaf $S$ of $\mathcal{S}$ intersects $D^{2} \times K$ in a single disc $F$, then for $i$ sufficiently large, there exists a subdisc $H_{i}$ of $D_{i}$ which is normally parallel to $S-\stackrel{\circ}{F}$, and $\partial H_{i} \subset \alpha_{i}$. This implies that $D_{i}$ is a sphere. Now suppose that some leaf $S$ of $\mathcal{S}$ intersects $D^{2} \times K$ in exactly 2-discs $F_{1}, F_{2}$. Given $n \in \mathbb{N}$, and an essential arc $\beta$ in the annulus $A-\stackrel{\circ}{F}_{1} \cup \stackrel{\circ}{F}_{2}$, then for $i$ sufficiently large, there exists a subdics $H_{i}$ of $D_{i}$ of the form $[0,1] \times[0, n]$ where $(\partial[0,1]) \times[0, n] \subset \alpha$, and $H_{i}$ naturally projects to $A$ as part of an $n$-fold cyclic cover. Finally each component of $[0,1] \times\{0, n\}$ naturally projects isomorphically onto $\beta$. Thus by coning off $H_{i} \cap \alpha_{i}$ one obtains a subdisc of $D_{i}$ whose boundary length is constant, independent of $i$, but whose area $\rightarrow \infty$ as $i \rightarrow \infty$. This thereby contradicts the least area property of $D_{i}$. A similar argument works when $S \cap D^{2} \times K$ has $r>2$ components. However the cases $r \leq 1$, together with $m(K)=1$, implies $\chi(\mathcal{S})=1$ and that the generic leaf of $\mathcal{S}-D^{2} \times K$ is an annulus thereby contradicting the $r=2$ argument.

Here is the idea behind what was used in the previous paragraph. Recall that we had, by Proposition 1.14, a sequence of measured branched surfaces $\left(B_{i}, m_{i}\right)$ which converged to a measured lamination with measure $m$, and that $\lambda$ was the minimal sublamination which supported $m$. Also $j>i$ implies that $\left(B_{j}, m_{j}\right)$ is obtained by splitting the measured branched surface $\left(B_{i}, m_{i}\right)$. Therefore if $T$ is a compact leaf of $\lambda$, then for $i$ sufficiently large $T$ embeds in $B_{i}$. If $r=1$, then $T$ is a disc so for $C$ sufficiently large, any $C$-splitting either isolates or destroys $T$. Since the latter does not occur, $T$ must appear as an isolated sector in $B_{j}$, for $j$ sufficiently large. If 
$r=2$, then $T$ is an annulus and a holonomy argument shows that for $i$ sufficiently large near $T \subset B_{i}$, part of each $D_{j}, j \geq i$, spirals around $T$. The spiraling must get arbitrarily large to obtain the annulus $T$ in the limit. Our $[0,1] \times[0, n]$ is a piece of that spiral. This completes the proof of Proposition 2.4

Proof of Theorem 2.1. Combine Propositions 2.4 and 1.14 with Theorem 1.16.

$\S 3$

\section{Hyperbolic Cellulations}

Definition 3.1. Let $\psi$ be a piecewise linear cellulation of the 3-manifold $M$. This means that $M$ is obtained by taking a disjoint union of 3-dimensional polyhedra $\sigma_{1}, \cdots, \sigma_{n}$ and pairwise identifying 2-dimensional faces in a PL fashion. Thus to each cell $C_{i}$ of $\psi$, there is a piecewise linear mapping $f_{i}: C_{i} \rightarrow M$ which restricts to

an embedding on $\stackrel{\circ}{C}_{i}$. We will usually suppress mentioning the $f_{i}$ 's and will denote the $i$-skeleton of $\psi$ by $\psi^{i}$.

As in the setting of triangulations define a normal curve (resp. local $\psi$-embedding) to be an immersion $f: \alpha \rightarrow \psi^{2}$ transverse to $\psi^{1}$, where $\alpha$ is a compact 1-manifold and for each 1-simplex $\eta$ in the induced triangulation on $\alpha, f \mid \alpha=f_{i} \circ t$, where $t: \eta \rightarrow C_{i}$ is an embedding into the 2-cell $C_{i}$ with $\partial \eta$ going to distinct edges (resp. $\eta$ not necessarily going to distinct edges.).

Similary define a normal disc to be a map $f: D^{2} \rightarrow M$, which factors through an embedding into a 3-cell $\sigma$ of $\psi$, so that $f \mid \partial D \rightarrow M$ is normal and (the lift into $\sigma$ ) crosses a given edge of $\sigma$ at most once. If $S$ is a surface, define a map $f:(S, \partial S) \rightarrow\left(M, \psi^{2}\right)$ to be normal if $f$ is transverse to $\psi^{2}, f \mid \partial S$ is a normal curve, and for each 2-cell $\eta$ in the induced cellulation on $S, f \mid S$ is a normal disc. If $S$ is a surface, define a map $f:(S, \partial S) \rightarrow\left(M, \psi^{2}\right)$ to be a-transverse to $\psi^{2}$ if $f$ is transverse to $\psi^{2}$ except at isolated points of $\partial S$ where $f$ appears as in Figure 1.6 and furthermore $f \mid \partial S$ is a local $\psi$-embedding. We say that $f: S \rightarrow N$ is normal, or $S$ is a normal surface, if $f$ is a branched immersion a-transverse to $\psi^{2}$, inducing a cellulation $\kappa$ on $S$ such that each 2-cell of $\kappa$ gets mapped to a normal disc in $\psi$. 
In particular $f \mid \partial S$ is a normal curve.

In order to suppress notation, we will often view $S$ as lying in $M$. E.g. if $Y$ is a submanifold of $M$ we may refer to $S \cap Y$ rather than $f^{-1}(Y)$.

If $\alpha$ is a curve in $\psi^{2}$ transverse to $\psi^{1}$, then define length $(\alpha)$ to be the number of 1 cells crossed by $\alpha$. If $f: S \rightarrow N$ is a-transverse to $\psi^{2}$, then $f$ induces a stratification $\kappa$ on $S$, where $f^{-1}\left(\psi^{i}\right)=\kappa^{i}$ the $i$-th strata. We'll abuse notation by calling a component of $\kappa^{i}-\kappa^{i-1}$ an $i$-cell. Finally define $\operatorname{area}(S)=\sum_{i=1}^{n} s\left(v_{i}\right) / \operatorname{valence}\left(f\left(v_{i}\right)\right)$. Here $v_{1}, \cdots, v_{n}$ are the vertices of $\kappa, s\left(v_{i}\right)$ is the local number of 2-cells of $\kappa$ touching $v_{i}$, and valence $\left(f\left(v_{i}\right)\right)$ is the local number of 2-cells of $\psi$ which come into $f\left(v_{i}\right)$. For example if $f: S \rightarrow M$ is an immersion of a closed surface, then $\operatorname{area}(S)$ is simply the intersection number of $S$ with $\psi^{1}$. This definition of area keeps track of the local branching at vertices and is additive under unions, e.g. if $S=S_{1} \cup S_{2}, S_{1} \cap S_{2} \subset \partial S_{1} \cap \partial S_{2}$, and $f \mid S$ is normal, then $\operatorname{area}\left(S_{1}\right)+\operatorname{area}\left(S_{2}\right)=$ $\operatorname{area}(S)$.

View the mass of $M$ as being concentrated near $\psi^{1}$. Think of a 1-cell $\phi$ as a $D^{2} \times I$. If $k 3$-cells of $\psi$ touch $\phi$, then pretend the $D^{2} \times I$ is subdivided into $k$ wedges, one for each 3-cell. Finally imagine that each $D^{2} \times t, t \in I$ is subdivided into $k$ pie slices, the area of each slice being $1 / k$.

We may use the notation length $\psi(\alpha)$ or $\operatorname{area}_{\psi}(D)$ to make clear that we are measuring with respect the cellulation $\psi$.

Lemma 3.2. If $f: S \rightarrow M$ is a mapping of a closed surface into the aspherical manifold $M$ with cellulation $\psi$, then $f$ can be homotoped to a normal surface, provided that for each essential simple closed curve in $S, 1 \neq\left[f_{*}(\alpha)\right] \in \pi_{1}(M)$ (i.e. $f$ injects on simple loops).

Proof. First homotope $f$ to a generic least area immersion transverse to $\psi^{2}$. After a further homotopy we can assume that the induced statification $\kappa$ is a cellulation. Among all such maps choose one that minimizes double points of $f \mid f^{-1}\left(\psi^{2}\right)$. If $\eta$ is a 2-cell of $\kappa$ which maps into the 3-cell $\sigma$, then $f \mid \partial \eta$ is an embedding (when lifted) into $\sigma$. Otherwise a homotopy of $f$ supported near $\eta$ reduces the number of double 
points. Thus we can assume that the restriction of $f$ to each 2-cell is an embedding (when lifted) into its associated 3-cell. Such an $f$ is normal.

Lemma 3.3. (Epstein - Penner [EP]). If $N$ is a complete noncompact hyperbolic 3-manifold of finite volume, then $N$ has a cellulation by ideal polyhedra.

Question 3.4. (Igor Rivin) Does every complete noncompact hyperbolic 3-manifold of finite volume have a cellulation by ideal triangles?

Definition 3.5. If the complete noncompact finite volume orientable hyperbolic 3manifold $N$ has a cellulation by ideal polyhedra, then removing a neighborhood of the ends of $N$ one obtains a compact manifold $M$ whose boundary is a non empty union of tori. The induced cellulation on $M$ is a cellulation by truncated ideal polyhedra. We call such a cellulation a relative hyperbolic cellulation. Call the newly created 2-cells (on $\partial M$ ) the facets.

Definition 3.6. Define a combinatorial geometry ( $\kappa$, angle) on the compact surface $S$ to be a cellulation $\kappa$ together with a function angle: $\{$ vertices of 2-cells of $\psi\} \rightarrow[0, \pi]$ such that if $v \in \kappa^{0}, w_{1}, \cdots, w_{n}$ are the vertices of 2-cells of $\kappa$ which are identified with the vertex $v$ of $\kappa$ and $\operatorname{Angle}(v) \stackrel{\text { def }}{=} \sum_{i=1}^{n} \operatorname{angle}\left(w_{i}\right)$, then $\operatorname{Angle}(v)=2 \pi$ for $v \in \stackrel{\circ}{S}$.

If $C$ is a 2-cell with vertices $v_{1}, \cdots, v_{n}$, then define $\int_{C} K=\sum_{i=1}^{n} \operatorname{angle}\left(v_{i}\right)-$ $(n-2) \pi=2 \pi-\sum_{i=1}^{n}\left(\pi-\operatorname{angle}\left(v_{i}\right)\right)$ and $\int_{S} K=\sum_{i=1}^{m} \int_{C_{i}} K$, where $C_{1}, \cdots, C_{m}$ are the 2-cells of $\kappa$. If $v$ is a vertex of $\kappa, v \in \partial S$, then define $\gamma_{g}(v)=\pi-\operatorname{Angle}(v)$ and $\int_{\partial S} \gamma_{g}=\sum_{v_{i} \in \kappa^{0} \cap \partial S} \gamma_{g}\left(v_{i}\right)$. Call a 2-cell $C$ respectively negatively curved, flat or positively curved if $\int_{C} K$ is respectively $<0,0$, or $>0$.

PL Gauss Bonnet theorem [We] 3.7. If $S$ has a combinatorial geometry $\left(\psi\right.$, angle), then $\int_{S} K+\int_{\partial S} \gamma_{g}=2 \pi \chi(S)$.

Remark 3.8. The proof is an elementary combinatorial exercise. See [We] for the case of a closed surface.

Remark 3.9. The following result is the obvious generalization of an observation of Andrew Casson which was stated for normal closed surfaces with respect to ideal 
triangulations on hyperbolic 3-manifolds.

Lemma 3.10. Let $\psi$ be a relative hyperbolic cellulation on $Y$. If $S$ is an immersed surface in $Y$ which is normal with respect to $\psi$. Then $f$ induces on $S$ a combinatorial geometry ( $\kappa$, angle) where $\kappa$ is the induced cellulation on $S$ and if $v \in \stackrel{\circ}{Y}$, then angle $(v)$ is the dihedral angle at the edge of the associated 3-cell of $\psi$. Otherwise $\operatorname{angle}(v)=\pi / 2$.

Corollary 3.11. (Casson [Ca]) Let $\psi$ be a relative hyperbolic cellulation on $Y$. If $S$ is an immersed closed surface in $Y$ which is is normal with respect to $\psi$, then $\chi(S) \leq 0$. Equality holds if and only if $S$ is a normally parallel to a peripheral torus.

Proof. Combine the the following result with Lemma 3.10 and Theorem 3.7.

Rivin's Lemma 3.12 [Ri]. If $\alpha$ is a normal closed curve on the boundary of an ideal hyperbolic polygon which crosses (not necessarily distinct) edges $e_{1}, \cdots, e_{n}$, then $2 \pi \leq \sum_{i=1}^{n}\left(\pi-d\left(e_{i}\right)\right)$, where $d\left(e_{i}\right)$ is the dihedral angle at $e_{i}$. Equality holds if and only if $\alpha$ is a simple closed curve which separates off a single facet from the rest.

Proof. Use the fact that any ideal polygon is the union of ideal tetrahedra and use the fact that for an ideal tetrahedron, dihedral angles of opposite edges are equal.

Remark 3.13. Its a famous theorem of Igor Rivin [Ri], that the converse is also true. That is any combinatorial geometry on a polygon satisfying the conclusion of Lemma 3.12 arises from a unique ideal hyperbolic polygon.

Remark 3.14. A closed surface of genus $g$ which is (Riemannian) least area in a hyperbolic 3 -manifold has area bounded above by $(2 g-2) \pi$. The rest of $\S 3$ is devoted to establishing a similar result for least area surfaces with respect to relative hyperbolic cellulations. Unlike the Riemannian setting, it may happen that $\int_{C} K>0$, where $C$ is a normal disc. However, we show that if $S$ is a normal surface in the relative hyperbolic cellulation, then after a small homotopy and redefinition 
of the combinatorial geometry, all such "positively curved" discs can be eliminated. It will then follow that the total integral over the negatively curved normal discs is bounded below by $2 \pi \chi(S)-\pi$ length $(\partial S)$. Therefore by the finiteness of normal disc types the negative curvature is concentrated in a finite area subsurface. After shoving, the flat part of $S$ out of $Y$, we are left with a surface $S_{Y}$ whose area is bounded above by $C_{0}$ ( length $\left.(\partial S)-\chi(S)\right)$ where $C_{0}$ is a constant depending only on $\psi$.

Definition 3.15. A compact immersed surface $S$ in the 3-manifold $Y$ with relative hyperbolic cellulation $\psi$ is $h$-normal if $S$ is a normal surface with respect to $\psi$ and satisfies the following additional properties.

i) $\partial S \cap \partial Y=\emptyset$

ii) $S$ has no fake boundary branching, and

iii) for each 1-cell $\beta$ in $\kappa$ (the induced cellulation on $S$ ) which lies in the 2-cell $\eta$ of $\psi$, if $\beta$ separates in $\eta$, a single vertex from the rest, then either $\beta \subset \partial Y$, or $\beta \cap \partial S, \neq \emptyset$. Call an arc of the latter type bad. See Figure 3.1.

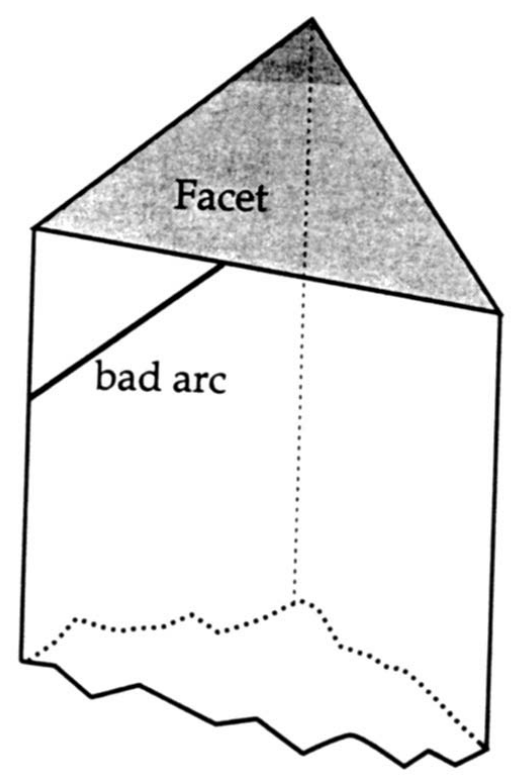

Figure 3.1 
The next lemma is a version of Lemma 3.2 for surfaces in relative hyperbolic cellulations. It says that at the cost of pushing part of $S$ out of $Y, S$ can be homotoped to be h-normal, without decreasing $\chi(S)$.

Lemma 3.16. Let $Y$ be a codimension-0 submanifold of the compact aspherical 3-manifold $M$ with $Y \cap \partial M=\emptyset$. Let $\psi$ be a relative hyperbolic cellulation on $Y$ and $f: S \rightarrow M$ satisfy $\partial S \subset \stackrel{\circ}{Y}, f \mid \partial S$ is normal, $f$ injects on simple loops, $f$ is transverse to $\psi^{2}$ near $\partial Y$ (and in particular transverse to $\partial Y$ ) and $f$ is boundary incompressible in the following sense. There is no properly embedded arc $\alpha \subset S \cap Y$ with endpoints in distinct 1-cells of the induced triangulation on $\partial S$, such that $f \mid \alpha$ can be homotoped rel $\partial \alpha$ into a 2-cell of $\psi$. Assume also that no component of $f^{-1}(Y)$ is a 2-disc disjoint from $\partial S$. Then $S$ can be homotoped rel $\partial S$ to $S_{1}$ such that $S_{1} \cap Y$ is h-normal respect to $\psi$ and $\chi\left(S_{1} \cap Y\right) \geq \chi(S \cap Y)$. Furthermore no component of $S_{1} \cap Y$ is a disc disjoint from $\partial S_{1}$.

Proof. Using the boundary incompressibility of $f$, first homotope $f$ rel $\partial S \cup(S \cap$ $\partial Y)$ to an immersion a-transverse to $\psi^{2}$. Homotope $f$ near $\partial S$ to eliminate fake boundary branching at the expense of introducing intersections as in Figure 1.6. If some component of $f^{-1}(Y)-f^{-1}\left(\psi^{2}\right)$ is not a disc, then $f$ can be homotoped rel $\partial S$ to reduce $\left|S \cap \psi^{1}\right|$. If for some 2-cell $C$ of $\kappa, f(\partial C)$ crossed a 1-cell $e$ of $\psi$ more than once, then a homotopy of $f$ reduces $\left|S \cap \psi^{1}\right|$. (But $\chi(S \cap Y)$ may rise if $e \subset \partial Y$.) If $S$ had a bad $\operatorname{arc} \beta$, then a small homotopy eliminates this arc thereby reducing $\left|S \cap \stackrel{\circ}{Y} \cap \psi^{1}\right|$ and possibly increasing $\left|S \cap \psi^{1}\right|$. Argue as in the proof of Lemma 3.2 to homotope $f$ so that the restriction of $f$ to each 2-cell of $\kappa$ is an embedding into its associated 3-cell. All of these homotopies can be accomplished without decreasing $\chi(S \cap Y)$ or introducing disc components of $S \cap Y$. The result follows by induction on $\left(\left|S \cap \stackrel{\circ}{Y} \cap \psi^{1}\right|,\left|S \cap \psi^{1}\right|\right)$ ordered lexicographically.

Proposition 3.17. Let $\psi$ be a relative hyperbolic triangulation on the compact 3manifold $Y$ which is contained in the interior of the compact 3-manifold $M$. Let $f$ : $S \rightarrow M$ be a map of a compact surface transverse to $\partial Y$ such that $f \mid \partial S \subset \stackrel{\circ}{Y}$. There exists a constant $C_{0}$ such that if $S \cap Y$ is an h-normal surface, then $S$ is homotopic 
rel $\partial S$ to a surface $S_{1}$, such that area $\left(S_{1} \cap Y\right) \leq C_{0}($ length $(\partial S)-\chi(S \cap Y))$.

Corollary 3.18. Under the hypothesis of Proposition 3.17, there exists a $C_{1}$, such that length $\left(\partial\left(S_{1} \cap Y\right)\right) \leq C_{1}($ length $(\partial S)-\chi(S \cap Y))$.

Proof. Apply the following Lemma 3.19 taking $C_{1}=v C_{0}$.

Remark. If $S$ is a Riemannian least area surface in $Y$ which goes far out the cusps, then the region out in the cusp accounts for little Riemannian area, but large PL area. The passage from $S$ to $S_{1}$ amounts to shoving the cusp stuff out of $Y$.

Lemma 3.19. Let $v$ be the maximal valence of an edge of $\psi$. If $T$ is a-transverse to $\psi$, then area $(T) \geq \operatorname{length}(\partial T) / v$.

Proof of Proposition 3.17. Let $\kappa$ be the induced cellulation on $S \cap Y$. Define a combinatorial geometry ( $\kappa$,angle) on $S \cap Y$ as follows. Let $v$ be a vertex of the 2-cell $\eta$ of $\kappa$.

If $f(v) \in \partial Y$, define angle $(v)=\pi / 2$.

If $v \in \partial S \cap \psi^{1}$, then define angle $(v)=0$.

If $v \in \partial S-\psi^{1}$, then define angle $(v)=\pi / 2$ (Such vertices arise as in Figure 1.6.)

Otherwise angle $(v)$ is the associated dihedral angle of the 3-cell $\sigma$ of $\psi$ which contains $\eta$.

Claim. If $\mathrm{C}$ is a 2-cell of $\kappa$, then $C$ is nonpositively curved. If $C$ is flat, then either

a) $f(C)$ is parallel to a facet and $C$ is disjoint from $\partial S$, or

b) $f(\partial C)$ encircles a vertex of $\psi$, or

c) $f(C)$ encircles a single edge $e$ on the boundary of the 3-cell which contains $f(C), e \not \subset \partial Y$ and $C \cap \partial S=\emptyset$. See Figure 3.2. 


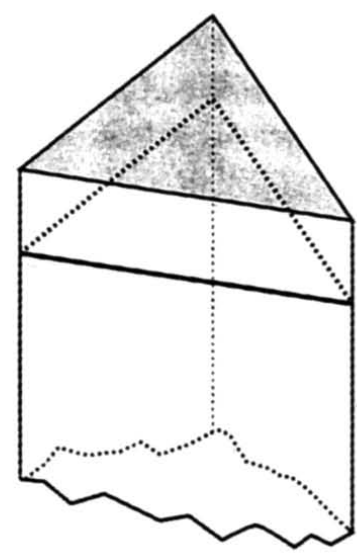

Type a)

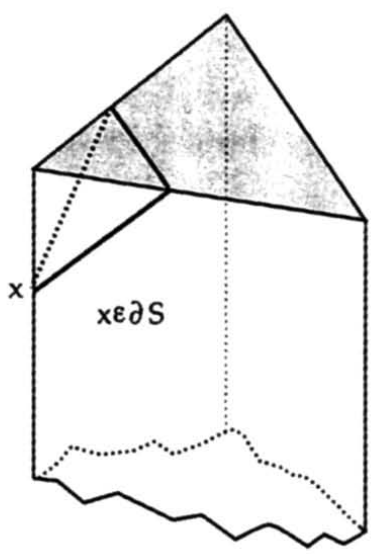

Type b)

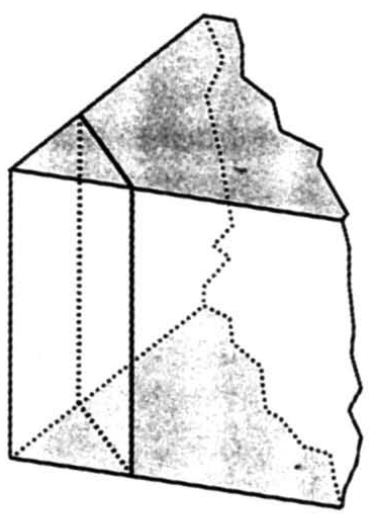

Type c)

Flat 2-cells

Figure 3.2

Proof of Claim. If $\sigma$ is the 3-cell which contains $f(C)$, then $f \mid C$ lifts to an embedding (also called $f$ ) of $C$ into $\sigma$. First suppose that $f(\partial C)$ is disjoint from the facets. Let $\hat{C}$ denote $C$ with the following combinatorial geometry. The 1-skeleton of $\hat{C}$ equals $\partial \hat{C}, f^{-1}\left(\psi^{1}\right) \cap C$ are the vertices of $\partial \hat{C}$, and each point $x$ of $f^{-1}\left(\psi^{1}\right)$ is assigned the dihedral angle of $\sigma$ at $f(x)$. Thus by Rivin's lemma, $\hat{C}$ is either negatively curved or is flat and parallel to a facet. If $C \cap \partial S=\emptyset$, then $C$ and $\hat{C}$ will have the same combinatorial geometry. If $C \cap \partial S \neq \emptyset$, then by comparing the combinatorial geometry of $\hat{C}$ with that of $C$ it follows that $\int_{C} K<\int_{\hat{C}} K \leq 0$.

If $\partial C$ crosses exactly one facet and has no bad subarcs, then by pushing $\partial C$ off of the facet, in at least one of the two possible ways, one obtains a new normal curve bounding a disc $C^{\prime}$ which is not more curved than $C$. Any two facets of a truncated polyhedron are connected by at most one edge. Thus if $C^{\prime}$ is flat, then $C$ is negatively curved. If $\partial C$ has a bad $\operatorname{subarc} \beta$, then by decree angle $(v)=0$ for the non facet vertex $v$ of $\partial \beta$. Another application of Rivin's Lemma implies that such a $C$ is flat if and only if $\partial C$ crosses exactly 3 edges of $\psi$.

If $\partial C$ crosses exactly two facets, and $C \subset \sigma$ is a 3-cell of $\psi$, then a similar argument to the one above shows that either $C$ is negatively curved or $\partial C$ separates off a single edge of $\sigma$ disjoint from $\partial S$. 
If $\partial C$ crosses more than two facets, then $C$ is negatively curved.

Even accounting for the possibility that part of a 2-cell lies in $\partial S$, there are only a bounded number of combinatorial possibilities for negatively curved h-normal 2cells in $\psi$. Thus there exists $c_{1}>0$ which depends only on $\psi$ such that $-c_{1}$ is the maximal possible value of $\int_{C} K$ where $C$ is a negatively curved h-normal 2-cell.

By definition for $v \in \kappa^{0} \cap \partial S$ either $f(v) \in \psi^{1}$ in which case $\gamma_{g}(v)=\pi$ or $f(v) \notin \psi^{1}$ in which case $\gamma_{g}(v)=0$. Also if $\alpha$ is a component of $f^{-1}(\partial Y)$, then $\int_{\alpha} \gamma_{g}=0$. Therefore $\int_{S} K=2 \pi \chi(S)-\pi \operatorname{length}(\partial S)$ and hence there are at most $\frac{1}{c_{1}}(\pi$ length $(\partial S)-2 \pi \chi(S))$ negatively curved 2-cells of $\kappa$.

Let $c_{2}$ be the maximal valence of an edge of $\psi$. The Claim and the fact that there is no fake boundary branching implies that there are at most $c_{2}$ length $(\partial S)$ flat 2-cells of type b). Since a flat 2-cell of type c) can only share an edge with either another type c) flat 2-cell or a negatively curved 2-cell, and that a string of $c_{2}$ type c) flat 2-cells leads to an area reducing homotopy of $S$, it follows that after homotopy there can be at most $\frac{c_{3} c_{2}}{c_{1}}(\pi$ length $(\partial S)-2 \pi \chi(S))$ type c) flat 2-cells. Here $c_{3}$ is the maximal length of the boundary of an h-normal 2-cell. Thus for some constant $c_{4}$, there are at most $c_{4}($ length $(\partial S)-\chi(S))$ 2-cells of $\kappa$ which are not flat of type a). Now let $W \subset S$ be the union of type a) flat 2-cells. Homotope $S$ to $S_{1}$ by a homotopy supported in $\stackrel{\circ}{W}$ to push most of $W$ out of $Y$. So if $T$ denotes $W$ with a small collar of $\partial W$ removed, then $S_{1} \cap Y=((S \cap Y)-\stackrel{\circ}{T}) \cup A$, where $A$ is a union of annuli connecting $\partial T$ straight to $\partial Y$. Therefore $\operatorname{area}\left(S_{1} \cap Y\right)<\operatorname{area}((S-\stackrel{\circ}{W}) \cap Y)+2 c_{2} \operatorname{length}(\partial W)<$ $c_{5}$ area $((S-\stackrel{\circ}{W}) \cap Y)<c_{5} c_{3} c_{4}($ length $(\partial S)-\chi(S))=C_{0}($ length $(\partial S)-\chi(S))$.

Proposition 3.20. Let $\psi$ be a relative hyperbolic triangulation on the compact 3manifold $Y \subset \stackrel{\circ}{M}$. Suppose that $f: S \rightarrow M$ is a map of a compact surface such that for each component $\delta$ of $\partial S$, either $\delta \cap Y=\emptyset$ or $f \mid \delta$ is an immersed curve in $\psi^{2} \cap \stackrel{\circ}{Y}$ which is transverse to $\psi^{1}, f$ injects on simple loops, $f$ is transverse to $\partial Y, f$ is a-transverse to $\psi^{2}$ and no component of $f^{-1}(Y)$ is a disc disjoint from $\partial S$. Then $S$ can be homotoped rel $\partial S$ to $S_{1}$ such that area $\left(S_{1} \cap Y\right) \leq \operatorname{Max}\left(0, C_{0}(\right.$ length $((\partial S) \cap$ $Y)-\chi(S \cap Y)))$. 
Proof of Proposition 3.20. The main improvement of this Proposition over the last is that $f: S \rightarrow M$ is not h-normal. We argue by induction on $c(S)=\operatorname{length}((\partial S) \cap$ $Y)-\chi(S \cap Y)$ and can assume that $S$ is connected. If $c(S)=-2$, then $S$ is a 2-sphere in $Y$ and can be homotoped out of $Y$. Now assume that the Proposition is true for $c(S)<k$. Using the induction hypothesis, its routine to verify the Proposition if $(\partial S) \cap Y$ is not a union of normal curves.

Suppose that there is an $\operatorname{arc} \alpha \subset f^{-1}(Y)$ with endpoints in distinct 1-cells of $\kappa$ such that $f \mid \alpha$ is homotopic into a 2-cell of $\psi$, via a homotopy fixing $\partial \alpha$ and supported in $Y$. Boundary compress along $\alpha$ to get a new map $f_{1}: T \rightarrow M$ which satisfies the hypothesis of the Proposition, and $c\left(f_{1}\right)<c(f)$. By induction, $f_{1}$ is homotopic rel $\partial T$ to $g_{1}$ which satisfies the conclusions of the Proposition. If $T_{1}$ is the resulting surface, then attach a little 1-handle to $T_{1}$ (missing $\psi^{1}$ ) to obtain a surface $S_{1}$ which is homotopic to $S$ rel $\partial S$ and satisfies the conclusion.

Therefore we can assume that $f$ satisfies the hypothesis of Lemma 3.16. Now apply Lemma 3.16 and Proposition 3.17.

Corollary 3.21. If $S_{1}$ is as in Proposition 3.20, then length $\left(\partial\left(S_{1} \cap Y\right)\right)$ $\leq \operatorname{Max}\left(0, C_{1}(\right.$ length $\left.((\partial S) \cap Y)-\chi(S \cap Y))\right)$.

Remark 3.22. The main results of $\S 1-2$ can all be stated in terms of cellulations rather than triangulations. In particular Proposition 1.14, Theorem 2.1 and Proposition 2.4 hold by substituting triangulation with cellulation. The proofs parallel those of $\S 1-2$. Of course a version of Proposition 1.10 also holds for cellulations, but there is not an obvious canonical branched surface which carries all normal laminations. For the sake of reference we single out

Proposition 3.23. Let $M$ be a closed 3-manifold with cellulation $\psi . \pi_{1}(M)$ is not negatively curved if and only if there exists a sequence of least area normal immersed discs $f_{i}: D_{i} \rightarrow M$, such that length $\left(\partial f_{i}\right) / \operatorname{area}\left(f_{i}\right) \rightarrow 0$ and no $f_{i}$ has fake boundary branching. 


\section{The UBIQUiTy ThEOREM}

Definition 4.1. If $\alpha$ is a homotopically trivial curve in the 3-manifold $M$ and $k \subset$ $M-\alpha$ is a smooth simple closed curve, then define the wrapping number $\operatorname{wr}(\alpha, k)=$ $\min \{|E \cap k|$ where $E \subset M$ is an immersed 2-disc transverse to $k$ such that $\partial E=\alpha\}$.

Theorem 4.2. Let $k$ be a smooth simple closed curve in the closed, irreducible 3manifold $M$ with cellulation $\psi$ such that $k$ is transverse to $\psi^{2}$, disjoint from $\psi^{1}$ and for each 2-cell $\eta \subset \psi,|k \cap \eta| \leq 1$. Then either $M$ is toroidal, or $k$ is contained in a 3-cell or there exists a constant $C$ such that for any least area disc $D$ a-transverse to $\psi^{2}$ with $\partial D \cap k=\emptyset$ we have area $(D) \leq C(w r(\partial D, k)+$ length $(\partial D))$.

Remark. Here length and area are measured as in Definition 3.1. Recall that normal immersed discs are examples of a-transverse discs.

The following result is an immediate consequence of Theorem 4.2.

Ubiquity Theorem 4.3. Let $k \not \subset B^{3}$ be a smooth simple closed curve in the closed, atoroidal, irreducible 3-manifold $M$ with cellulation $\psi$ such that $k$ is transverse to $\psi^{2}$, disjoint from $\psi^{1}$ and for each 2-cell $\eta \subset \psi,|k \cap \eta| \leq 1$. There exists constants $K$ and $L$ such that if $D$ is a least area disc a-transverse to $\psi^{2}$ with $\partial D \cap k=\emptyset$ and length $(\partial D) / \operatorname{area}(D)<L$, then $w r(\partial D, k) / \operatorname{area}(D)>K$.

Corollary 4.4. Let $\lambda$ be a quasi-least area semi-Euclidean lamination in the closed atoroidal irreducible 3-manifold $M$. If $k \subset M-\lambda$ is a smooth simple closed curve, then $k$ lies in a 3-cell.

Proof of Corollary 4.4. Suppose that $\lambda$ is normal with respect to the cellulation $\psi$ and all measurements are taken with respect to $\psi$. After passing to a subdivision of $\psi$ and isotopy of $k$ we can assume that for each 2-cell $\eta$ of $\psi$ that $|k \cap \eta| \leq 1$. Note that the property of a leaf being conformally Euclidean or quasi-least area is preserved after passing to subdivision. If $L$ is a conformally Euclidean leaf of $\lambda$, then by the Ahlfors Lemma, [Ca] p. 499, there exists a sequence of embedded discs $\tilde{E}_{1} \subset \tilde{E}_{2} \subset \cdots \subset \tilde{L}$ the universal covering of $L$, such that length $\left(\partial \tilde{E}_{i}\right) / \operatorname{area}\left(\tilde{E}_{i}\right)<$ 
$1 / i$. Since $\lambda$ is quasi least area there exists a constant $K_{1}$ such that for each $i$, $\operatorname{area}\left(\tilde{E}_{i}\right) \leq K_{1}$ area $\left(D_{i}\right)$ where $D_{i}$ is a least area disc in $\tilde{M}$ with $\partial \tilde{E}_{i}=\partial D_{i}$. If $E_{i}$ is a slightly perturbed projection of $\tilde{E}_{i}$ to $M$ such that $\partial E$ is embedded, then $\operatorname{wr}\left(\partial E_{i}, k\right)=0$ all $i$. Also if $k \not \subset B^{3}$, and $i$ is sufficiently large then we obtain a contradiction to Theorem 4.3 .

Remarks 4.5. i) In words the Ubiquity Theorem says that the area of a least area disc $D$ of small isoperimetric ratio is proportional to the wrapping number of $\partial D$ and $k$.

ii) The proof we give relies on Thurston's hyperbolization theorem for Haken 3-manifolds. One can give a much less elegant proof which uses only [Gr] and standard 3-manifold topology.

Proof of Theorem 4.2. To obtain a clue, read the proof of case 1. For a complete and more detailed argument read the proof of case 2 .

Case 1. $M-k$ is hyperbolic.

Proof of Case 1. It suffices to consider the case that $\psi$ is obtained by attaching a 2-cell and a 3 -cell to a relative hyperbolic cellulation on $M-\stackrel{\circ}{N}(k)$. And it suffices to prove Theorem 4.2 for $\psi$-least area discs $D$ such that $\partial D \subset M-N(k)$. Given such a disc $D$, let $E \subset M$ be an immersed disc such that $\partial E=\partial D$ and $\operatorname{wr}(\partial D, k)=|E \cap k|=|E \cap \partial N(k)|$. By Proposition 3.20 and Corollary $3.21 E$ can be homotoped to a disc $E_{1}$ rel $\partial E$, such that area $(D) \leq \operatorname{area}\left(E_{1}\right)=\operatorname{area}\left(E_{1} \cap(M-\right.$ $\stackrel{\circ}{N}(k)))+\operatorname{area}\left(E_{1} \cap N(k)\right) \leq \operatorname{area}\left(E_{1} \cap(M-\stackrel{\circ}{N}(k))\right)+\operatorname{length}\left(\partial E_{1} \cap N(k)\right) \leq\left(C_{0}+\right.$ $\left.C_{1}\right)($ length $(\partial E)-\chi(E \cap(M-\stackrel{\circ}{N}(k))))=\left(C_{0}+C_{1}\right)($ length $(\partial D)+\operatorname{wr}(\partial D, k)-1)<$ $\left(C_{0}+C_{1}\right)($ length $(\partial D)+\operatorname{wr}(\partial D, k))$.

Case 2. General case

\section{Proof of Case 2.}

If $k$ does not lie in a 3 -cell, then $M-\stackrel{\circ}{N}(k)$ is irreducible and hence there exists by the characteristic manifold theory of [JS], [J] a collection of $\pi_{1}$-injective pair wise disjoint embedded tori $T_{0}, T_{1}, \cdots, T_{n} \subset M$ such that each component of 
$(M-\stackrel{\circ}{N}(k))-\cup_{i=0}^{n} T_{i}$ is either a finite volume hyperbolic 3-manifold or the interior of a graph manifold. (Recall that all 3-manifolds in this paper are orientable and that a graph manifold is the union of finitely many Seifert fibred spaces glued together along some of their boundary components.) Here $T_{0}=\partial N(k)$. By Thurston [Th] $M-k$ is hyperbolic if and only if $\left\{T_{0}, T_{1}, \cdots, T_{n}\right\}=\left\{T_{0}\right\}=\{\partial N(k)\}$ and $M-\stackrel{\circ}{N}(k)$ is a not a graph manifold.

If $T$ is an embedded $\pi_{1}$-injective non boundary parallel torus in $M-\stackrel{\circ}{N}(k)$, then either $T$ is $\pi_{1}$-injective in $M$ and hence Theorem 4.2 is proved or $T$ is compressible. By standard 3-manifold topology, if $T$ is compressible, then either $T$ bounds a unique solid torus $W$ in $M$ or $T$ bounds a cube with knotted hole $W$. In the former case $k \subset W$. In the latter case there exists an embedded $B^{3} \subset M$ and a knotted properly embedded arc $\beta \subset B^{3}$ such that $W=B^{3}-\stackrel{\circ}{N}(\beta)$ and thus $k \cap W=\emptyset$. Note that $k \not \subset B^{3}$ implies that $T$ cannot bound both a solid torus and a cube with knotted hole. For each $i$, let $W_{i}$ be the associated solid torus or cube with knotted hole bounded by $T_{i}$. Observe that if $T_{i} \subset \stackrel{\circ}{W}_{j}$, then $W_{i} \subset \stackrel{\circ}{W}_{j}$.

The $W_{i}$ 's are partially ordered by inclusion, so reorder the $T_{i}$ 's so that $W_{0}$ is the maximal solid torus region (which is necessarily nonempty since for some $\left.i, W_{i}=N(k)\right)$ and $W_{1}, \cdots W_{r}$ are the maximal cube with knotted hole regions. Let $Y=M-\stackrel{\circ}{W}_{0} \cup \cdots \cup \stackrel{\circ}{W}_{r}$.

Claim 1. Either Theorem 4.2 is true or $Y$ is atoroidal and hence by Thurston [Th] has a complete hyperbolic metric of finite volume.

Proof of Claim 1. By construction $Y$ is either atoroidal or is a graph manifold. We will assume the latter and derive a contradiction. If $r>0$, then Figure 4.1 shows how to reembed $Y \cup W_{0} \cup \cdots \cup W_{r-1}$ into $M$ in such a way that $\partial W_{r}^{\prime}$ bounds a solid torus $V_{r}$ in $M-Y^{\prime} \cup W_{0}^{\prime} \cup \cdots \cup W_{r-1}^{\prime}$, where $W_{i}^{\prime}$ (resp. $\partial W_{r}^{\prime}, Y^{\prime}$ ) denotes the reembedded $W_{i}$ (resp. $\left.\partial W_{r}, Y\right)$. There are an infinite number of such reembeddings, by "twisting the neck". Now fix a graph structure on $Y$. This structure induces a Seifert fibering on $\partial W_{r}$. At most one reembedding corresponds to spanning Seifert fibres of $\partial W_{r}^{\prime}$ by meridinal discs of $V_{r}$. After any other reembedding, $M-W_{0}^{\prime} \cup$ 
$\cdots \cup W_{r-1}^{\prime}$ is a graph manifold. By repeating this operation $r-1$ more times, we conclude that $M$ is obtained by attaching a solid torus to the boundary of a graph manifold. Since such a manifold is either reducible, has finite $\pi_{1}$ or has a $\pi_{1}$-injective immersed torus, Claim 1 is established. Note that if $\pi_{1}(M)$ is finite, then $M$ has a linear isoperimetric inequality.

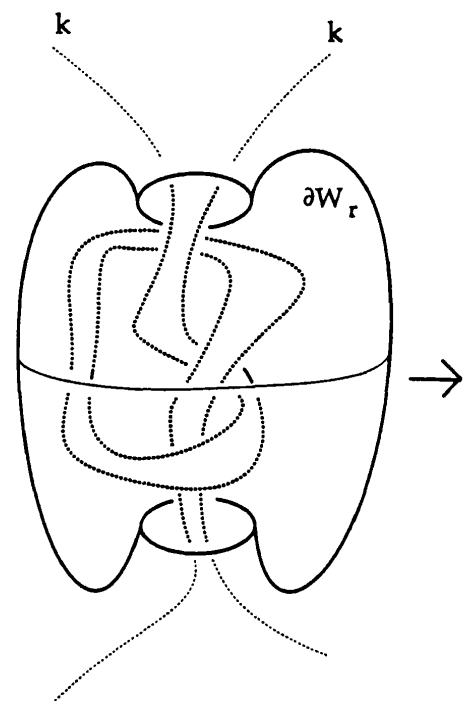

$\partial \mathrm{W}$ does not bound a solid torus

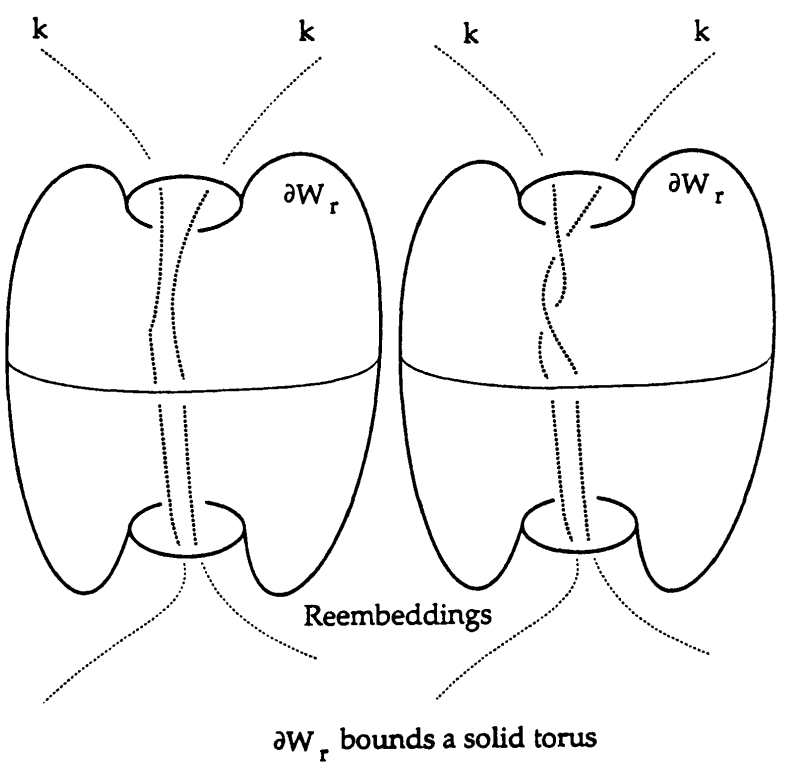

Figure 4.1

Given a $\psi$-least area disc $D$, define

$$
W_{\psi}(D, k)=\frac{\operatorname{wr}(\partial D, k)+\text { length }_{\psi}(\partial D)}{\operatorname{area}_{\psi}(D)} .
$$

We may suppress the subscript $\psi$ when the cellulation is understood.

Claim 2. We can assume that $\psi$ satisfies

a) $\psi \mid Y$ is a relative hyperbolic cellulation and in particular for each $i \leq r, \psi$ restricts to a cellulation on $W_{i}$.

b) There exists a $D^{2} \times S^{1} \subset \stackrel{\circ}{W}_{0}(k)$ called $N_{1}(k)$ such that $k \subset \stackrel{\circ}{N}_{1}(k)$ is a core of $N_{1}(k), \psi^{2} \cap N_{1}(k)$ consists of $\partial N_{1}(k)$ and a finite number of pairwise disjoint meridinal discs. Furthermore if $\sigma$ is a 3-cell of $\psi \mid N_{1}(k)$, then $k \cap \sigma$ is a properly embedded unknotted arc. Note that $\psi^{1} \cap \stackrel{\circ}{N}_{1}(k)=\emptyset$. See Figure 4.2 


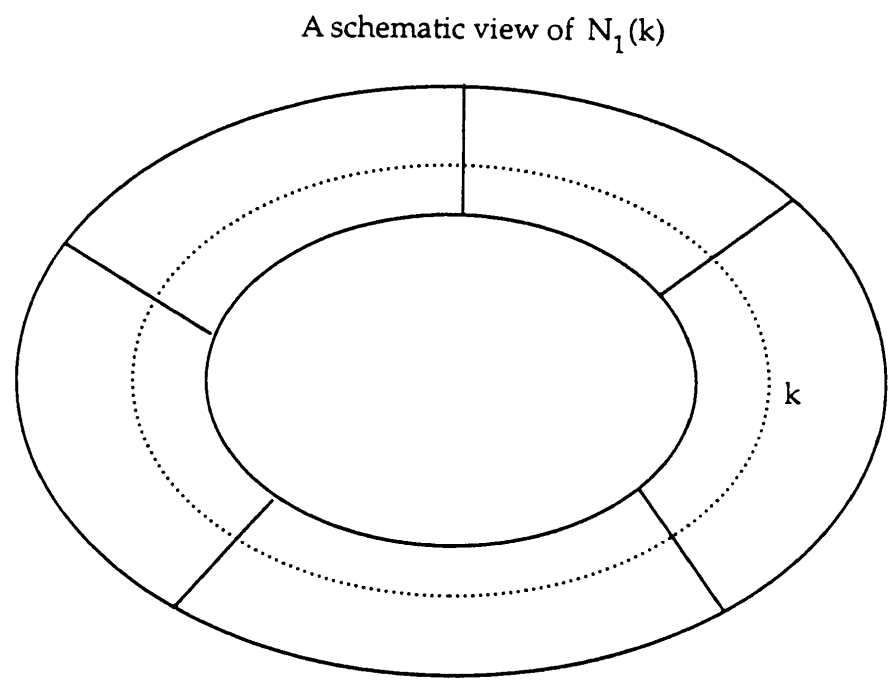

Figure 4.2

Proof of Claim 2. If $\phi$ is a subdivision of $\psi$ and Theorem 4.2 holds for $\phi$, then Theorem 4.2 holds for $\psi$. Use the fact that there exists a $K_{1}>0$ such that if $D$ is a $\psi$-least area disc, then there is a $\psi$ least area disc, also called $D$, with $W_{\psi}(D, k)$ unchanged such that $D$ is a $K_{1}$-quasi $\phi$-least area disc. Furthermore length $_{\psi}(\partial D) \leq \operatorname{length}_{\phi}(\partial D) \leq K_{1}$ length $_{\psi}(\partial D)$. The last inequality uses the fact that an embedded arc $\alpha$ in a 2-cell of $\psi$ is isotopic rel $\partial \alpha$ to an $\operatorname{arc}$ crosing $\phi^{1}$ a uniformly bounded number of times, via an isotopy disjoint from $k$. (This is where we use the hypothesis that for each 2-cell $\eta$ of $\psi,|k \cap \eta| \leq 1$.) Thus a sequence $\left\{D_{i}\right\}$ of $\psi$-least area discs with $\mathrm{W}_{\psi}\left(D_{i}\right) \rightarrow 0$ gives rise to a sequence $\left\{E_{i}\right\}$ of $\phi$-least area discs with $W_{\phi}\left(E_{i}\right) \rightarrow 0$.

Conversely suppose that $\phi$ is a subdivision of $\psi$ such that if $\sigma$ is a 3-cell of $\psi$ and $\sigma \cap k \neq \emptyset$, then $\phi|\sigma=\psi| \sigma$. Then standard arguments show that there exists a $K_{2} \geq 1$ such that a $\phi$-least area disc $D$ give rise to a $\psi$-least area disc $E$ where $\frac{\text { length }_{\psi}(\partial E)}{\operatorname{area}_{\psi}(E)} \leq \frac{K_{2} \text { length }_{\phi}(\partial D)}{\operatorname{area}_{\phi}(D)}$ and $\partial E$ is homotopic to $\partial D$ via a homotopy disjoint from $k$. Thus if Theorem 4.2 holds for $\psi$, then it holds for $\phi$. Similarly this result follows in the following case. Here if $\sigma$ is a 3-cell of $\psi$, then either $k \cap \sigma=\emptyset$ or $k \cap \sigma$ 
is an unknotted arc and $\phi$ is a subdivision of $\psi$ such that $\phi^{2} \cap k=\psi^{2} \cap k$.

Given $\psi$, we first construct a subdivision $\psi_{1}$ with the property that for each 3-cell $\sigma$ of $\psi_{1}, k \cap \sigma=\emptyset$ or $k \cap \sigma$ is an unknotted arc. The subdivision is created on each 3-cell B of $\psi$ as follows. Let $t=B \cap k$. Think of $B$ as $D^{2} \times I$ with $\partial t \subset D^{2} \times 0$ and $t$ in generic position with respect to the height function coming from the $I$-factor. Now attach a finite number of pairwise disjoint properly embedded 2-cells $C_{1}, \cdots, C_{n} \subset B$, one for each local minimum of $t \subset \stackrel{\circ}{B}$, with the property that for each $i, C_{i}$ separates off a 3 -cell $B_{i}$ with $B_{i} \cap t$ is an unknotted arc and $t \cap\left(B-\cup_{i=1}^{n} \stackrel{\circ}{B}_{i}\right)$ is a trivial $B^{3}$-link on $s$ components. Now subdivide each $C_{i}$ along a properly embedded arc such that each "half" of of $C_{i}$ hits $t$ exactly once. See Figure 4.3. After subdividing along another $s-12$-cells we obtain a cell division on $B$ such that each 3-cell intersects $t$ in an unknotted arc. $\psi_{1}$ is the result of these $n+s-1$ subdivisions of $\psi$. One readily checks in $n+s-1$ steps that Theorem 4.2 holds for $\psi$ if and only if it holds for $\psi_{1}$.

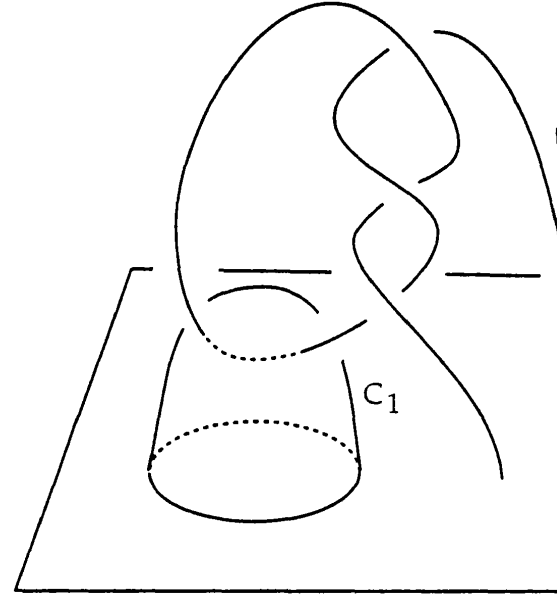

a)

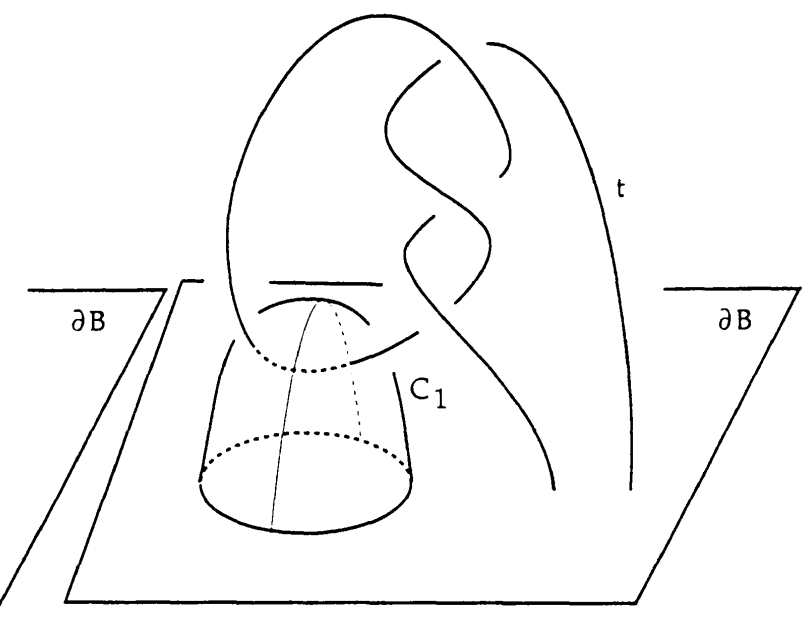

Figure 4.3

b)

Let $N_{1}(k)$ be a $D^{2} \times S^{1}$ in $\stackrel{\circ}{W}_{0}$ whose core is $k$ such that $N_{1}(k) \cap \psi_{1}^{1}=\emptyset$ and $N_{1}(k) \cap \psi_{1}^{2}$ is a disjoint union of meridinal discs. Subdivide $\psi_{1}$ to $\psi_{2}$ so that $N_{1}(k) \cap \psi_{2}^{2}=N_{1}(k) \cap \psi_{1}^{2} \cup \partial N_{1}(k)$. By the second paragraph of this proof, Theorem 4.2 holds for $\psi_{2}$ if and only if it holds for $\psi_{1}$.

By $[\mathrm{EP}]$ there exists a hyperbolic cellulation on $Y$ and hence there exists a 
cellulation $\psi_{3}$ on $M$ satisfying a) and b) and which agrees with $\psi_{2}$ near $N_{1}(k)$. By the uniqueness of PL structures for 3 -manifolds [Mo], there exists a cellulation $\psi_{4}$ on $M$ isomorphic to $\psi_{3}$ via an isomorphism which restricts to the identity on $N_{1}(k)$ such that $\psi_{4}$ and $\psi_{2}$ have a common subdivision $\psi_{5}$ and $\psi_{5}\left|N_{1}(k)=\psi_{4}\right| N_{1}(k)=$ $\psi_{2} \mid N_{1}(k)$. By the first two paragraphs Theorem 4.2 holds for $\psi_{4}$ if and only if it holds for $\psi_{5}$ if and only if it holds for $\psi_{2}$. Therefore Theorem 4.2 holds for $\psi_{4}$ if and only if it holds for $\psi$. Since $\psi_{4}$ satisfies the conclusions of Claim 2 , that result follows.

Claim 3. It suffices to prove Theorem 4.2 for $\operatorname{discs} D$ such that $\partial D \subset \stackrel{\circ}{Y}$.

Proof of Claim 3. It suffices to prove Theorem 4.2 for discs $D$ such that $\partial D \cap$ $N_{1}(k)=\emptyset$. Indeed by construction of $N_{1}(k)$, a small homotopy disjoint from $k$, pushes $\partial D$ off of $N_{1}(k)$ and so $D$ gives rise to a least area disc $E$ a-transverse to $\psi$ with $E \cap N_{1}(k)=\emptyset, \operatorname{wr}(\partial E, k)=\operatorname{wr}(\partial D, k)$ and $D, E$ have approximate isoperimetric inequalities.

Since balls and solid tori have linear isoperimetric inequalities it follows that there exists a $K_{3}$ such that if $\gamma$ is a locally $\psi$-embedded homotopically trivial curve in $M-N_{1}(k)$ then there exists a homotopy $H: S^{1} \times I \rightarrow M$ of $\gamma$ to $\gamma_{1}$ such that $\gamma_{1}$ is locally $\psi$-embedded, $\gamma_{1} \subset \stackrel{\circ}{Y}$ and area $(H) \leq K_{3}$ length $(\gamma)$. This implies that if $D$ (resp. $E$ ) is a least area disc spanning $\gamma$, (resp. $\gamma_{1}$ ), then

$$
\begin{gathered}
\operatorname{area}(E) \geq \operatorname{area}(D)-\operatorname{area}(H) \\
\operatorname{length}\left(\gamma_{1}\right) \leq \operatorname{length}(\gamma)+v(\operatorname{area}(H)) \leq\left(1+v K_{3}\right) \operatorname{length}(\gamma) \\
\operatorname{wr}\left(\gamma_{1}, k\right) \leq \operatorname{wr}(\gamma, k)+3 \operatorname{area}(H) \leq \operatorname{wr}(\gamma, k)+3 K_{3} \operatorname{length}(\gamma)
\end{gathered}
$$

The first and third inequalities are immediate. The second follows from Lemma 3.19. To obtain the fourth, note that $H$ can be chosen so that if $\eta$ is a 2-cell in the induced cellulation on $S^{1} \times I$, and $H(\eta) \cap \stackrel{\circ}{N}_{1}(k) \neq \emptyset$, then $H \mid \eta$ is an embedding and $H(\eta) \cap \psi^{1} \neq \emptyset$. (This uses the fact $\gamma \cap N_{1}(k)=\emptyset$.) Since $H$ can be chosen so that $|\eta \cap k| \leq 1$ and $\operatorname{area}(H \mid \eta) \geq \frac{1}{3}$ it follows that $\left|H^{-1}(k)\right| \leq 3 \operatorname{area}(H)$. 
Thus a sequence of discs $\left\{D_{i}\right\}$ such that $W\left(D_{i}, k\right) \rightarrow 0$ gives rise to a sequence $\left\{E_{i}\right\}$ with $W\left(E_{i}, k\right) \rightarrow 0$ and $\partial E_{i} \subset \stackrel{\circ}{Y}$.

Let $E$ be an immersed disc in $M$ a-transverse to $\psi^{2}$ such that $\partial E=\partial D$ and $|E \cap k|=w r(\partial D, k)$. Among all such discs with these properties choose one that minimizes $|\partial Y \cap E|$. Let $E_{Y}=E \cap Y$. Assume that length $(\partial E)>0$.

Claim 4. $-\chi\left(E_{Y}\right)<\operatorname{wr}(\partial E, k)$

Proof of Claim 4. Since $Y$ has no essential annuli, and $|\partial Y \cap E|$ is minimal, each component of $E_{Y}$ disjoint from $\partial E$ is a disc with at least two open discs removed. Define a partial ordering on the components $F_{1}, \cdots, F_{m}$ of $E_{Y}$, by $F_{i}<F_{j}$ if $F_{i}$ is separated from $\partial E$ by $F_{j}$. Let $\tilde{F}_{i}=\cup_{F_{j} \leq F_{i}} F_{j}$ and $\hat{F}_{i}$ be the subdisc of $E$ bounded by the outermost component of $\partial F_{i}$. Since for some $i, E_{Y}=\tilde{F}_{i}$ and $E=\hat{F}_{i}$ it suffices to show that for each $i,-\chi\left(\tilde{F}_{i}\right) \leq\left|\hat{F}_{i} \cap k\right|-1$. To prove this let $F_{i}$ be a minimal component of $E_{Y}$. All but one component of $\partial F_{i}$ bounds a disc in $E$ whose interior is disjoint from $F_{i}$. Let $G$ be one such disc. The incompressibility of $\partial W_{i}$ in $W_{i}, i>0$ implies that $G \subset W_{0}$. The incompressibility of $\partial W_{0}$ in $W_{0}-k$ implies that $G \cap k \neq \emptyset$. Hence $-\chi\left(F_{i}\right)<\left|\hat{F}_{i} \cap k\right|$. Now inductively assume that for all $F_{i}<F_{j},-\chi\left(F_{i}\right) \leq\left|\hat{F}_{i} \cap k\right|-1$. Again let $G \subset E$ be the disc spanning an innermost component of $\partial F_{j}$. By either the induction hypothesis, if $\stackrel{\circ}{G} \cap E_{Y} \neq \emptyset$ or the previous argument, if $\stackrel{\circ}{G} \cap E_{Y}=\emptyset$ it disjoint from $\partial E$ follows that $-\chi\left(G \cap E_{Y}\right) \leq|G \cap k|-1$ and therefore $-\chi\left(\tilde{F}_{j}\right) \leq\left|\hat{F}_{j} \cap k\right|-1$.

Claim 5. $E$ can be homotoped to $E^{\prime}$ rel $\partial E$ such that if $F$ is the component of $E_{Y}^{\prime}$ which contains $\partial E$, then

1) $\operatorname{area}(F)<C_{0}(\operatorname{wr}(\partial D, k)+$ length $(\partial D))$ and

2) length $(\partial F)<C_{1}(\operatorname{wr}(\partial D, k)+$ length $(\partial D))$.

Proof. Let $S$ be the compact codimension-0 submanifold of $E$ such that $S \cap Y$ is exactly the component of $E \cap Y$ which contains $\partial E$. By Proposition 3.20 and Claim 4, $S$ can be homotoped rel $\partial S$ to $S_{1}$ such that area $\left(S_{1} \cap Y\right) \leq C_{0}(\operatorname{length}((\partial S) \cap$ $Y)-\chi(S \cap Y))<C_{0}($ length $(\partial E)+\operatorname{wr}(\partial E, k))$. Since the homotopy of $S$ to $S_{1}$ 
extends trivially to a homotopy of $E$ and $\partial E=\partial D$ conclusion 1) follows. (Here $E^{\prime}$ is the homotoped $E$ and $F$ is the component of $S_{1} \cap Y$ containing $\partial E$.) Conclusion 2) follows from Proposition 3.21.

Claim 6. There exists a constant $k_{2}$ such that if $\alpha$ is a closed curve in $W_{i}$, which is homotopically trivial in $M$, then $\alpha$ bounds a disc $A$ such that $\operatorname{area}(A)$ $<k_{2}$ (length $\left.(\alpha)\right)$.

Proof. Since balls and solid tori have linear isoperimetric inequalities and each $W_{i}, i \geq 1$, lies in a 3 -cell, Claim 6 holds (using the same constant $k_{3}$ ) for all $\alpha \subset W_{i}, i \geq 1$, and for all $\alpha \subset W_{0}$ which are homotopically trivial in $W_{0}$.

Let $\beta \subset W_{0}$ be a normal curve which generates $\pi_{1}\left(W_{0}\right)$. There exists a $k_{4}$ such that if $\alpha \subset W_{0}$ is not null homotopic in $W_{0}$, then $\alpha$ is homotopic in $W_{0}$ to $\beta^{n}, n \neq 0$ via a homotopy $L: S^{1} \times I \rightarrow M$ such that $\operatorname{area}(L)+|n| \leq k_{4} \operatorname{length}(\alpha)$. Since $\alpha$ is homotopically trivial in $M, \beta^{n}$ and hence $\beta$ (since $\pi_{1}(M)$ is torsion free) are homotopically trivial in $M$. If $B$ (resp. $A$ ) is a least area disc bounded by $\beta$ (resp. $\alpha)$, then

$$
\begin{aligned}
\operatorname{area}(A) \leq & |n| \operatorname{area}(B)+\operatorname{area}(L) \leq(1+\operatorname{area}(B))(|n|+\operatorname{area}(L)) \\
& \leq 2(1+\operatorname{area}(B)) k_{4} \operatorname{length}(\alpha)=k_{5} \operatorname{length}(\alpha),
\end{aligned}
$$

where $k_{5}=(1+\operatorname{area}(B)) k_{4}$. Finally, take $k_{2}=\max \left(k_{3}, k_{5}\right)$.

To complete the proof of Theorem 4.2 observe that $\partial D$ bounds a disc $J$ which is the union of $F$ (which was defined in Claim 5) and least area discs bounded by $\partial F-\partial D$. Thus by Claims 5 and 6 we obtain $\operatorname{area}(D) \leq \operatorname{area}(J) \leq \operatorname{area}(F)+$ $k_{2}$ length $(\partial F) \leq\left(C_{0}+C_{1} k_{2}\right)(\operatorname{wr}(\partial D, k)+$ length $(\partial D))$.

\section{$\S 5$}

\section{Ubiquity In The Smooth Category}

Theorem 5.1. Let $k$ be a smooth simple closed curve in the closed irreducible Riemannian 3-manifold $M$. Then either $M$ is toroidal or $k$ is contained in a 3-cell 
or there exists a constant $C>0$ such that if $D$ is a least area disc with $\partial D \cap k=\emptyset$, then area $(D) \leq C(w r(\partial D, k)+\operatorname{length}(\partial D))$.

Proof: Step 1. Either $k \subset B^{3}$ or $M$ is toroidal or $M=Y \cup W_{0} \cup \cdots \cup W_{r}$ where $W_{0}$ is a $D^{2} \times S^{1}, k \subset \stackrel{\circ}{W}_{0}, \partial W_{0}$ is $\pi_{1}$-injective in $W_{0}-k, W_{i} \cap W_{j}=\emptyset$ if $i \neq j$, and if $i \geq 1$, then $W_{i}$ lies in a 3 -cell and $\partial W_{i}$ is a torus which is $\pi_{1}$-injective in $W_{i}$. Furthermore $Y \neq \emptyset$ and $\stackrel{\circ}{Y}$ has a hyperbolic structure of finite volume. Finally $Y \cap\left(W_{0} \cup \cdots \cup W_{r}\right)=\partial Y$.

Proof of Step 1. For the proof of step 1, read (in §4) from the beginning of the proof of case 2, through the end of the proof of Claim 1.

Step 2. It suffices to establish Theorem 5.1 for any Riemannian metric on $M$.

Proof of Step 2. Use the fact that changes in the Riemannian metric changes arc length as well as area by uniformly bounded multiplicative amounts.

Let $X$ be a space diffeomorphic to $\stackrel{\circ}{Y}$ and give $X$ a complete hyperbolic metric. Let $T \times[0, \infty)$ cut off the ends of $X$ and be parametrized so that each $T \times i$ is a union of $r+1$ horospherical tori and for $z \in T, z \times[0, \infty)$ is a geodesic parametrized by arc length.

Let $N(\partial Y) \subset\left(W_{0} \cup \cdots \cup W_{r}\right)-k$ be a closed regular neighborhood of $\partial Y$. Let $g$ be a Riemannian metric on $M$ such that $g \mid Y$ is isometric to $X-T \times(0, \infty)$ and $g \mid N(\partial Y)$ is isometric to $T \times[0,1]$. I.e. $g \mid Y \cup N(Y)$ is the pullback metric induced from a diffeomorphism $f: Y \cup N(Y) \rightarrow X-T \times(1, \infty)$ such that $f(Y)=X-T \times(0, \infty)$ and $f(N(\partial Y))=T \times[0,1]$.

Step 3. a) There exists $\gamma>0$ with the following property. It suffices to prove Theorem 5.1 for discs $D$ where the geodesic curvature at each point of $\partial D \cap Y$ is bounded above by $\gamma$.

b) We can assume that $\partial D \cap N(\partial Y)$ is a union of curves of the form $z \times[0,1]$.

c) We can assume the $\partial D$ is embedded in $M$.

Let $D$ be a least area disc in $M$ such that $\partial D \cap k=\emptyset$. Let $E$ be a smooth disc which spans $\partial D$, is transverse to $k \cup N(\partial Y),|E \cap k|=\operatorname{wr}(\partial D, k)$ and satisfies the 
following two conditions.

i) No component of $E \cap Y$ disjoint from $\partial E$ is either a disc or an annulus

ii) If $\alpha \subset E \cap Y$ is a simple closed curve, then either $\alpha$ bounds a disc in $E \cap Y$ or $\alpha$ is homotopically nontrivial in $Y$.

Remark. The reader may have noticed that we have abused notation by identifying the disc $E$ with its image in $M$ thereby suppressing the mapping $f: E \rightarrow M$ which defines that image. Thus it would have been more precise to have first defined $f: E \rightarrow M$ and then rephrased i) by "no component of $f^{-1}(Y)$ is a disc or annulus disjoint from $\partial E$ " and ii) by "If $\alpha$ is a simple closed curve in $f^{-1}(Y)$, then either $\alpha$ bounds a disc in $f^{-1}(Y)$ or $f \mid \alpha$ is homotopically nontrivial as a map into $Y$. In the following statement $E \cap Y$ denotes a subset of the disc $E$.

Step 4. $-\chi(E \cap Y)<\operatorname{wr}(\partial E, k)$

Proof of Step 4. By hypothesis $\operatorname{wr}(\partial E, k)=|E \cap k|$. Define a partial ordering on the components $F_{1}, \cdots, F_{m}$ of $E \cap Y$, by $F_{i}<F_{j}$ if $F_{i}$ is separated from $\partial E$ by $F_{j}$. Let $\tilde{F}_{i}=\cup_{F_{j} \leq F_{i}} F_{j}$ and $\hat{F}_{i}$ be the subdisc of $E$ bounded by the outermost component of $\partial F_{i}$. Since $E \cap Y$ is the union of $\tilde{F}_{i_{1}}, \cdots, \tilde{F}_{i_{n}}$ where $F_{i_{1}}, \cdots, F_{i_{n}}$ are the maximal components, it suffices to show that for each $j,-\chi\left(\tilde{F}_{i_{j}}\right) \leq\left|\hat{F}_{i_{j}} \cap k\right|-1$. The proof of this fact is given after Claim $4 \S 4$. Ignore the assertion, true only in $\S 4$, that $\partial E=\partial F_{i}$ for some i. In that proof $E_{Y}$ denotes $E \cap Y$. [Note that for each $i$, no component of $\hat{F}_{i} \cap Y$ is a disc or annulus disjoint from $\partial E$, each disc component of $\hat{F}_{i} \cap W_{j}$ disjoint from $\partial E$ must lie in $W_{0}$ and nontrivially intersect $k$, and that $\left.\left.\operatorname{wr}\left(\partial \hat{F}_{i}, k\right)=\left|\hat{F}_{i} \cap k\right| \cdot\right)\right]$

Step 5. There exists constants $C_{4}, C_{5}$ such that after homotopy of $E$ rel $\partial E$, there exists a codimension-0 submanifold $H$ of $E$ such that $E \cap Y \subset H \subset Y \cup N(\partial Y)$ and

a) $\operatorname{area}(H) \leq C_{4}($ length $(\partial E)+\operatorname{wr}(\partial E, k))$

b) length $(\partial H) \leq C_{5}$ (length $\left.(\partial E)+\operatorname{wr}(\partial E, k)\right)$

Proof of Step 5. Let $H_{1}=E \cap Y$ and view $H_{1}$ as lying in $X-T \times(0, \infty)$. After a 
homotopy of $E$ rel $\partial E$, supported near $\partial Y$ we can assume that $H_{1} \cap T \times 0$ is straight in the Euclidean tori $T \times 0$. Let $H_{2} \subset X$ be $H_{1} \cup\left(\left(H_{1} \cap T \times 0\right) \times[0, s]\right)$. [More precisely, if $f: H_{1} \rightarrow Y$, then define $H_{2}=H_{1} \cup\left(f^{-1}(\partial Y) \times[0, s]\right) / \sim$ where $x \in f^{-1}(\partial Y)$ is identified with $x \times 0$. Finally extend $f$ to $H_{2}$ by $f(x, t)=(f(x), t) \in T \times t$.] Choose $s$ sufficiently large so that, length $\left(H_{2} \cap(T \times s)\right)<\frac{1}{2}$. After an extremely tiny perturbation of $\partial H_{2}$ near $T \times s$ we can assume that $\partial H_{2}$ is embedded in $X$. By Theorem 3 of Meeks - Yau [MY] (generalizing [SY]) $H_{2}$ is homotopic rel $\partial H_{2}$ (in $X$ ) to a least area surface $H_{3}$. It follows that for $s$ sufficiently large we obtain

$$
\begin{aligned}
\operatorname{area}\left(H_{3}\right) & =\int_{H_{3}} d A \\
& \leq \int_{H_{3}}-K d A \\
& =-2 \pi \chi\left(H_{3}\right)+\int_{\partial H} \gamma_{g} \\
& \leq-2 \pi \chi\left(H_{3}\right)+\gamma \operatorname{length}\left(\partial H_{3} \cap Y\right)+\pi|\partial E \cap(M-\stackrel{\circ}{Y})|+\frac{1}{2} \\
& <2 \pi w r(\partial E, k)+\gamma \operatorname{length}((\partial E \cap Y))+\frac{\pi}{2} \operatorname{length}(\partial E \cap N(\partial Y)) \\
& \leq C_{4}(\operatorname{length}(\partial E)+\operatorname{wr}(\partial E, k))
\end{aligned}
$$

where $C_{4}=\max (2 \pi, \gamma)$. The second equation follows from the fact that $H_{3}$ is a minimal surface and hence its sectional curvature is $\leq-1$ everywhere. The third equation is Gauss - Bonnet. To establish the fourth equation use the fact that $\partial H_{3} \cap Y$ has geodesic curvature bounded above by $\gamma, \partial H_{3} \cap T \times[0, s)$ is geodesic and hence has zero geodesic curvature and $\partial H_{3}$ near $T \times s$ is essentially horospherical, hence has geodesic curvature equal extremely close to 1 . Finally note that there are two right angled corners of $\partial H_{3}$ for each arc component of $\left(\partial H_{1}\right) \cap \partial Y$. The number of such components is equal to $|\partial E \cap(M-\stackrel{\circ}{Y})|$. To obtain the fifth equation observe that $-\chi\left(H_{3}\right)=-\chi\left(H_{2}\right)=-\chi\left(H_{1}\right)=-\chi(E \cap Y)<\operatorname{wr}(\partial E, k)-\frac{1}{2}$, the last inequality following by Step 4 . Also each component of $\partial E \cap(M-\stackrel{\circ}{Y})$ corresponds to two components of $\partial E \cap N(\partial Y)$ each of which has length 1.

After a small perturbation of $H_{3}$ we can assume that the conclusion of the above series of equations still hold and except for finitely many levels, $H_{3}$ is 
transverse to each $T \times i$. Since $\int_{0}^{1} \operatorname{length}\left(H_{3} \cap(T \times t)\right) d t \leq \operatorname{area}\left(H_{3} \cap(T \times\right.$ $[0,1])) \leq \operatorname{area}\left(H_{3}\right)$, it follows that for some $t \in[0,1), H_{3}$ is transverse to $T \times t$ and length $\left(H_{3} \cap(T \times t)\right) \leq \operatorname{area}\left(H_{3}\right) \leq C_{4}($ length $(\partial E)+\operatorname{wr}(\partial E, k))$. Let $H=$ $H_{3} \cap X-T \times(t, \infty)$. Thus area $(H) \leq \operatorname{area}\left(H_{3}\right) \leq C_{4}(\operatorname{length}(\partial E)+\operatorname{wr}(\partial E, k))$ and length $(\partial H) \leq \operatorname{length}(\partial E)+\operatorname{length}\left(H_{3} \cap T \times t\right) \leq 2 C_{4}($ length $(\partial E)+\operatorname{wr}(\partial E, k))$. Let $C_{5}=2 C_{4}+1$. To complete the proof of Step 5, view $H$ as lying in $Y \cup N(\partial Y)$ and observe that there is a homotopy of $E$ rel $\partial E$ supported in $Y \cup N(\partial Y)$ such that after the homotopy $E \cap(Y \cup T \times[0, t])=H$.

Proof of Theorem 5.1. Let $G$ be the closure of $E-H$. By the proof of Step 5 length $(\partial G) \leq \operatorname{length}(\partial E)+\operatorname{length}\left(H_{3} \cap T \times t\right) \leq C_{5}($ length $(\partial E)+\operatorname{wr}(\partial E, k))$. Each component of $\partial G \subset W_{i}$ for some i and is homotopically trivial in $M$. The argument of Claim $6(\S 4)$ shows that there exists a constant $C_{6}$ such that if $\alpha$ is a closed curve in some $W_{i}$ and $\alpha$ is homotopically trivial in $M$, then $\alpha$ bounds a $\operatorname{disc} D_{\alpha} \subset M$ such that $\operatorname{area}\left(D_{\alpha}\right) \leq C_{6}$ length $(\alpha)$. Therefore we can span $\partial G$ by a collection of $\operatorname{discs} \mathcal{D}$ such that $\operatorname{area}(\mathcal{D}) \leq C_{6} \operatorname{length}(\partial G)$.

One can construct a disc $F$ spanning $\partial D$ by piecing together a subset of the components of $H$ together with a subset of the least area discs bounded by components of $\partial G$. In fact, use those components of $H$ which, as subsets of $E$, nontrivially intersect $\partial E$ and those components of $\partial G$ which are outermost in $E$. It follows that $\operatorname{area}(D) \leq \operatorname{area}(F) \leq \operatorname{area}(\mathcal{D} \cup H) \leq \operatorname{area}(H)+C_{6} \operatorname{length}(\partial G) \leq$ $\left(C_{4}+C_{6} C_{5}\right)($ length $(\partial E)+\operatorname{wr}(\partial E, k))$.

Ubiquity Theorem 5.2. Let $k \not \subset B^{3}$ be a smooth simple closed curve in the closed, atoroidal, irreducible 3-manifold $M$. There exists constants $K$ and $L$ such that if $D$ is a least area disc with $\partial D \cap k=\emptyset$ and length $(\partial D) / \operatorname{area}(D)<L$, then $w r(\partial D, k) / \operatorname{area}(D)>K$.

Corollary 5.3. Let $\lambda$ be a quasi-least area semi-Euclidean lamination in the closed atoroidal irreducible 3-manifold $M$. If $k \subset M-\lambda$ is a smooth simple closed curve, then $k$ lies in a 3-cell.

Remark 5.4. By defining $\operatorname{wr}(\alpha, k)$ for all reasonable homotopically trivial curves $\alpha$ 
(rather than just $\alpha$ with $\alpha \cap k=\emptyset$ ) in the appropriate manner we can then obtain $5.1,5.2,5.3$ with the hypothesis $\partial D \cap k=\emptyset$ eliminated.

$\S 6$ A Very Brief Survey On The Geometrization Of 3-Manifolds

Thurston Geometization Conjecture 6.1 [Th]. Let $M$ be a closed connected irreducible 3-manifold, then either

a) $M=S^{3} / \Gamma$ where $\Gamma \subset \operatorname{Isom}\left(S^{3}\right)$ or

b) $\mathbb{Z} \oplus \mathbb{Z} \subset \pi_{1}(M)$ or

c) $M=\mathbb{H}^{3} / \Gamma$ where $\Gamma \subset \operatorname{Isom}\left(\mathbb{H}^{3}\right)$

Remarks 6.2. i) These conclusions exactly parallel the conclusions of the corresponding geometrization theorem for closed surfaces.

ii) The three conclusions are mutually exclusive. Conclusion a) is the geometrization conjecture for 3-manifolds with finite fundamental group. That conjecture is equivalent to positive solutions to each of the following longstanding open problems.

Poincare Conjecture 6.3. If $M$ is a closed connected simply connected 3-manifold, then $M$ is homeomorphic to $S^{3}$.

Generalized Space Form Conjecture 6.4. If the finite group $\Gamma$ acts freely on $S^{3}$, then $\Gamma$ is conjugate to a linear action.

Remark 6.5. See [Liv], [My], [Ru], and [T] for partial results on this conjecture. In particular by [T], Conjecture 6.4 for cyclic groups implies a positive solution to the following classical question.

Space Form Conjecture 6.6. If $M$ is covered by $S^{3}$, then $\pi_{1}(M)$ is isomorphic to a subgroup of $S O(4)$

As of this writing there is one class of groups that still needs to be eliminated [Mi], [Lee].

Remark 6.7. Conclusions b) and c) of the Geometrization conjecture treat the case that $\pi_{1}(M)$ is infinite. The topology of closed irreducible 3-manifolds with $\mathbb{Z} \oplus \mathbb{Z} \subset$ $\pi_{1}(M)$ is now completely understood, thanks to the work of [CJ], [G2], [M], and 
[Sc1-2]. These works in turn built on ideas of many others e.g. Tukia, Ghering, Martin, Jaco, Shalen, Johannson, Waldhausen, Burde, Zieschang, Neuwirth, and Murasugi. (See [G2] for a more detailed history.) Such manifolds contain either embedded $\pi_{1}$-injective surfaces or are Seifert fibred spaces. The [JS][J]-theory, discussed in $\S 4$, details the structure of 3 -manifolds with $\pi_{1}$-injective embedded tori. The nature of 3-manifolds $M$ with $\mathbb{Z} \oplus \mathbb{Z} \subset \pi_{1}(M)$ was not known when the Geometrization conjecture was originally formulated and conclusion b) was spelled out with all its possibilities.

Conclusion c) is also known as the

Thurston Hyperbolization Conjecture 6.8. Let $M$ be a closed, connected, irreducible 3-manifold with $\pi_{1}(M)$ infinite and $\mathbb{Z} \oplus \mathbb{Z} \not \subset \pi_{1}(M)$, then $M=\mathbb{H}^{3} / \Gamma$ where $\Gamma \subset \operatorname{Isom}\left(\mathbb{H}^{3}\right)$.

Remark 6.9. In 1978 Thurston proved this conjecture for Haken 3-manifolds [Th]. A result known in those days as the Monster Theorem.

At the JDG 96 conference, the author announced the following result [GMT] which was joint with Robert Meyerhoff and Nathaniel Thurston.

Theorem 6.10. Let $N$ be a closed hyperbolic 3-manifold. Then

a) If $f: M \rightarrow N$ is a homotopy equivalence where $M$ is an irreducible 3-manifold, then $f$ is homotopic to a homeomorphism.

b) If $f, g: M \rightarrow N$ are homotopic homeomorphisms, then $f$ is isotopic to $g$.

c) The space of hyperbolic metrics on $N$ is path connected.

Remark 6.11. i) The analogue of a) for spherical manifolds is false. Its a classical theorem of Reidemeister and Whitehead that the lens space $L(7,1)$ is homotopy equivalent but not homeomorphic to $L(7,2)$. Its a much more recent observation of the author that there exists an orientation preserving self homotopy equivalence of a lens space which is not homotopic to a homeomorphism [G3]. For example, consider the homotopy equivalence of $L(8,1)$ to itself whose action on $\pi_{1}$ is multiplication by 3 . 
ii) Theorem 6.10 reduces the Hyperbolization Conjecture to

Conjecture 6.12. If $M$ is a closed, connected, aspherical 3-manifold with $\mathbb{Z} \oplus \mathbb{Z} \not \subset$ $\pi_{1}(M)$, then $M$ is homotopy equivalent to a hyperbolic 3-manifold. Equivalently [since $M$ is a $K(\pi, 1)], \pi_{1}(M)$ is isomorphic to $\pi_{1}(N)$ where $N$ is a hyperbolic 3-manifold.

Conjecture 6.12 is equivalent to the following two well known open problems.

Group Negative Curvature Conjecture 6.13. If $M$ is a closed, connected, aspherical 3-manifold and $\mathbb{Z} \oplus \mathbb{Z} \not \subset \pi_{1}(M)$, then $\pi_{1}(M)$ is negatively curved.

See $[\mathrm{Gr}],[\mathrm{Bu}],[\mathrm{Sch}],[\mathrm{Mol}],[\mathrm{MO}]$, and $[\mathrm{Ka}]$ for some contributions towards Conjecture 6.13.

Cannon Conjecture 6.14. If $M^{3}$ is closed, connected, aspherical and $\pi_{1}(M)$ is negatively curved, then $\pi_{1}(M)$ is isomorphic to the fundamental group of a closed hyperbolic 3-manifold.

It was first proved by Casson [Ca] and Poenaru [Po], that if $M$ is closed, aspherical with a negatively curved fundamental group, then the universal covering of $M$ is $\mathbb{R}^{3}$. Also by [HRS] and [M] a closed irreducible 3-manifold $M$ with $Z \oplus Z \subset \pi_{1}(M)$ is covered by $\mathbb{R}^{3}$. Thus a special case of Conjecture 6.13 is the longstanding

Conjecture 6.15. If $M$ is a closed irreducible connected 3-manifold with $\pi_{1}(M)$ infinite, then $M$ is covered by $\mathbb{R}^{3}$.

Remarks 6.16. i) See p. 149-151 [Ki] for a list of contributions to this problem.

ii) Bestvina - Mess $[\mathrm{BM}]$ show that the sphere at infinity of an aspherical 3manifold with negatively curved fundamental group is a 2-sphere.

Remark 6.17. See [Can], and [CFP] for some very remarkable and beautiful developments towards Jim Cannon's program to establish Conjecture 6.14. In particular the introduction to [CFP] gives a lucid overview of both the general program and the state of their work. 


\section{REFERENCES}

[BM] M. Bestvina \& J. Mess, The Boundary of Negatively Curved Groups, J. AMS 4, 469-481.

[Bo] B. Bowditch, Notes on Gromov's Hyperbolicity Criterian for Path-Metric Spaces, Group Theory From a Geometrical Viewpoint (Trieste 1990), World Sci. (1991), 64-167.

[Bu] S. V. Buyalo, Euclidean Planes in 3-Dimensional Manifolds of Non Positive Curvature, Math. Notes 43 (1988), 60-66.

[C] A. Casson, U. Montreal Lecture June 1995.

[Can] J. Cannon, The Combinatorial Riemann Mapping Theorem, Acta. Math. 173 (1994), 155-234.

[Ca] A. Candel, Uniformization of Surface Laminations, Ann. Ecole Norm. Sup. 26 (1993), $489-516$.

[CFP] J. Cannon \& W. Floyd \& W. Parry, Sufficiently Rich Families of Planar Rings, preprint.

[Ch] J. Christy, Immersing Branched Surfaces in Dimension-3, Proc. AMS 115 (1992), 853861 .

[CJ] A. Casson \& D. Jungreis, Convergence Groups and Seifert Fibered 3-Manifolds, Invent. Math. 118, 441-456.

[Co] A. Connes, A survey on Foliations and Operator Algebras, Proc. Symp. Pure Math. 38 (1982), 521-628.

[EP] D. Epstein \& R. Penner, Euclidean Decompositions of non Compact Hyperbolic Manifolds, J. Diff. Geom 27 (1988), 67-80.

[FO] W. Floyd \& U. Oertel, Incompressible Surfaces via Branched Surfaces, Topology 23, 117-125.

[G1] D. Gabai, The Simple Loop Conjecture, J. Diff. Geom 22 (1985), 143-149.

[G2] Convergence Groups are Fuchsian Groups, Ann. Math. 136 (1992), 447-510.

[G3] On the Geometric and Topologica Rigidity of Hyperbolic 3-Manifolds, Bull. AMS 31 (1994), 228-232.

[G4] - Problems in the Geometric Theory of Foliations and Laminations on 3-Manifolds, AMS/IP Stud. Adv. Math, 2.2 (1997), 1-33.

[GK] D. Gabai \& W. H. Kazez, Group Negative Curvature for 3-Manifolds with Genuine Laminations, Geom. Top., http://www.maths.warwick.ac.uk/gt/, 2 (1998), 65-77.

[GMT] D. Gabai \& R. Meyerhoff \& N. J. Thurston, Homotopy Hyperbolic 3-Manifolds are Hyperbolic, MSRI-preprint.

[GO] D. Gabai \& U. Oertel, Essential Laminations in 3-Manifolds, Ann. Math. (2) 130 (1989), 41-73.

[Gh] E. Ghys, Gauss Bonnet Theorem for 2-Dimensional Foliations, J. Funct. Anal. 77 (1988), 51-59.

[Gr] M. Gromov, Hyperbolic Groups, MSRI Pubs. 8, 75-264.

[GS] S. Gerston and J. Stallings, Casson's Idea about 3-Manifolds whose Universal Cover is $\mathbb{R}^{3}$, Int. J. Alg. Comp. 1 (1991), 395-406.

[Ha] W. Haken, Theorie der Normal Flachen, Acta. Math. 105 (1961), 245-375.

[HRS] J. Hass \& H. Rubinstein \& G. P. Scott, Compactifying Coverings of Closed 3-Manifolds, J. Diff. Geom. 30, 817-832.

[J] K. Johannson, Homotopy Equivalences of 3-Manifolds with Boundary, Springer LNM 761 (1979).

[JS] W. Jaco \& P. Shalen, Seifert Fibered Spaces in 3-Manifolds, Mem. AMS 21 (1979).

[Ka] M. Kapovich, Flats in 3-Manifolds, preprint.

[Kl] B. Kleiner, in preparation.

[Lee] R. Lee, Semicharacteristic Classes, Topology 12 (1973), 183-199.

[Liv] G. R. Livesay, Fixed Point Free Involutions on the 3-Sphere, Ann. of Math. 59 (1960), $603-611$.

[LS] R. Lyndon \& P. Schupp, Combinatorial Group Theory, Erge. der Math., Springer 89 (1977).

[M] E. Moise, Affine Structures on 3-Manifolds V, Ann. Math. (2) 56 (1952), 96-114.

[Me] J. Mess, The Seifert Fibred Space Conjecture and Groups Coarse Quasiisometric to Planes, preprint.

[Mi] J. Milnor, Groups which Act on $S^{n}$ without Fixed Points, Am. J. Math. 79 (1957), 623630 .

[Mo] E. Moise, Affine Structures in 3-Manifolds V, Ann. Math. 56 (1952), 96-114. 
[Mo1] L. Mosher, Geometry of Cubulated 3-Manifolds, Topology 34 (1995), 789-814.

[MO] L. Mosher \& U. Oertel, Spaces which are not Negatively Curved, Comm. Anal. Geom. 6 (1998), 67-140.

[MO1] , Two-dimensional Laminations of Positive Euler-Characteristic, to appear, Quart. J. Math.

[My] R. Myers, Free Involutions on Lens Spaces, Topology 20, 313-318.

[MY] W. Meeks \& S. T. Yau, The Equivariant Loop Theorem for Three-Dimensional Manifolds and a Review of the Existence Theorems for Minimal Surfaces, Academic Press, The Smith Conjecture (1984), 153-163.

[Pa] P. Papasoglu, On the Sub-Quadratic Isoperimetric Inequality, Ohio St. U. math. Res. Inst. Publ. 3 (1995), 149-157.

[Pl] J. Plante, Foliations with Measure Preserving Holonomy, Ann. Math. (2) 102 (1975), 327-361.

[Po] V. Poenaru, Almost Convex Groups, Lipshitz Combing and $\pi_{1}^{\infty}$ for various Universal Covering Spaces of Closed 3-Manifolds, J. Diff. Geom. 35 (1992), 103-130.

[Po1] V. Poenaru, The Collapsible Pseudo-Spine Representation Theorem, Topology 31 (1992), 625-656.

[Ri] I. Rivin, A Characterization of Ideal Polyhedra in Hyperbolic 3-Space, Ann. Math. (2) 143 (1996), 51-70.

[Ru] H. Rubinstein, 1996 CBMS Lecture Notes.

[Sc1] G. P. Scott, A New Proof of the Annulus and Torus Theorems, Amer. J. Math. 2 (1978), 241-277.

[Sc2] $\quad$ There are no fake Seifert Fibred Spaces with Infinite $\pi_{1}$, Ann. Math. 117 (1985), 35-70.

[Sch] V. Schroeder, Codimension-1 Tori in Manifolds of Non Positive Curvature, Geom. Ded. 33 (1990), 251-263.

[SY] R. Schoen \& S. T. Yau, Existence of Incompressible Minimal Surfaces and the Topology of Three Dimensional Manifolds with Non-Negative Scalar Curvature, Ann. Math. 110 (1979), 127-142.

[T] C. B. Thomas, Elliptic Structures on 3-Manifolds, LMS lecture notes 104 (1986).

[Th] W. P. Thurston, Three-Dimensional Manifolds, Kleinian Groups and Hyperbolic Geometry, Proc. Symp. Pure Math. 39 (1979), 87-111.

[We] J. Weeks, The Shape of Space, Pure and Applied Math. 96, Marcel Dekker (1985).

California Institute of Technology, Pasadena, CA 91125-0001 USA

E-mail address: gabai@its.caltech.edu 